\title{
Three-dimensional macroporous nanoelectronic networks as minimally invasive brain probes
}

\section{Citation}

Xie, Chong, Jia Liu, Tian-Ming Fu, Xiaochuan Dai, Wei Zhou, and Charles M. Lieber. 2015. “ThreeDimensional Macroporous Nanoelectronic Networks as Minimally Invasive Brain Probes." Nat Mater 14 (12) (October 5): 1286-1292. doi:10.1038/nmat4427.

\section{Published Version}

doi:10.1038/nmat4427

\section{Permanent link}

http://nrs.harvard.edu/urn-3:HUL.InstRepos:24981602

\section{Terms of Use}

This article was downloaded from Harvard University's DASH repository, and is made available under the terms and conditions applicable to Other Posted Material, as set forth at http:// nrs.harvard.edu/urn-3:HUL.InstRepos:dash.current.terms-of-use\#LAA

\section{Share Your Story}

The Harvard community has made this article openly available.

Please share how this access benefits you. Submit a story.

\section{Accessibility}




\section{Three-dimensional macroporous nanoelectronic networks as minimally-invasive brain probes}

Chong Xie ${ }^{1}$, Jia Liu ${ }^{1}$, Tian-Ming Fu ${ }^{1}$, Xiaochuan Dai ${ }^{1}$, Wei Zhou ${ }^{1}$ and Charles M.

Lieber $^{1,2 *}$

${ }^{1}$ Department of Chemistry and Chemical Biology, and ${ }^{2}$ School of Engineering and Applied

Sciences, Harvard University, Cambridge, Massachusetts, 02138, USA.*e-mail:

cml@cmliris.harvard.edu 
Abstract. Direct electrical recording and stimulation of neural activity using microfabricated silicon and metal micro-wire probes have contributed extensively to basic neuroscience and therapeutic applications; however, the dimensional and mechanical mismatch of these probes with the brain tissue limits their stability in chronic implants and decreases the neuron-device contact. Here, we demonstrate the realization of a 3D macroporous nanoelectronic brain probe that combines ultra-flexibility and subcellular feature sizes to overcome these limitations. Built-in strains controlling the local geometry of the macroporous devices are designed to optimize the neuron/probe interface and to promote integration with the brain tissue while introducing minimal mechanical perturbation. The ultra-flexible probes were implanted frozen into rodent brains and used to record multiplexed local field potentials (LFPs) and single-unit action potentials from the somatosensory cortex. Significantly, histology analysis revealed filling-in of neural tissue through the macroporous network and attractive neuron-probe interactions, consistent with long-term biocompatibility of the device.

Currently, there is intense interest in the development of materials and electronic devices that can extend and/or provide new capabilities for probing neural circuitry and afford long-term minimally-invasive brain-electronics interfaces ${ }^{1}, 2,3,4$. Conventional brain probes have contributed extensively to basic neuroscience ${ }^{5,6}$ and therapeutic applications ${ }^{7,8,9,10}$, although they suffer from chronic stability and poor neuron-device contacts ${ }^{4,11,12,13}$. Recent studies of smaller $^{14,15}$ and more flexible ${ }^{16,17}$ probes suggest that addressing size and mechanical factors could help overcome current limitations. 
The most common neural electrical probes are fabricated from metal ${ }^{18}$ and silicon ${ }^{19,20}$, materials that have very different structural and mechanical properties compared to brain tissue ${ }^{21}$. Evidence suggests that mechanical mismatch is an important reason leading to abrupt and chronically unstable interfaces within the brain $^{4,22}$. For example, motion of skull-affixed rigid probes in chronic experiments can induce shear stresses and lead to tissue scarring ${ }^{13,23}$, and thereby compromise the stability of recorded signals on the time scale of weeks to months ${ }^{4,24,25}$. More recent work has shown that flexible probes fabricated on polymer substrates ${ }^{12,17}$ and smaller-sized probes ${ }^{11,14}$ can reduce deleterious tissue response. More generally, there has also been effort developing flexible bioelectronics ${ }^{26,27,28}$ and nanoscale devices for single cell recording ${ }^{29,30}$. We have also shown that $3 \mathrm{D}$ macroporous electronic device arrays can function as a scaffold for and allow for 3D interpenetration of cultured neuron cell networks without an adverse effect on cell viability ${ }^{31}$, and such networks can be injected by syringe through needles into materials, including brain tissue ${ }^{32}$. In the latter case, it remains challenging to make electrical input/output (I/O) connections needed for recording signals since the conventional I/O cannot pass through the injection needles.

Taking the above facts into consideration, we define an ideal implantable neural probe as (i) possessing a stiffness similar to brain tissue to minimize/eliminate mechanically-induced scarring, (ii) a high-degree of porosity and cellular/subcellular feature sizes to allow for interpenetration and integration of neurons and neural projections with the electronics, (iii) a means for implantation of the resulting extremely flexible structure, and (iv) facile $\mathrm{I} / \mathrm{O}$ to allow for multiplexed recording. Our strategy to meet these constraints focuses on implementing 3D macroporous nanoelectronic networks ${ }^{31,33}$, where the macroporous nanoelectronic probe has a mesh-like structure designed to promote interpenetration and close integration with neural tissue 
(Figs. 1a,b). The mesh design is unique in having a two-dimensional (2D) open area of ca. $80 \%$, feature sizes to sub-10 micron scale, and importantly, a high flexibility with effective bending stiffness of $<0.64 \times 10^{-15} \mathrm{~N} \cdot \mathrm{m}^{2}$ (Supplementary Information) 4-7 orders of magnitude smaller than conventional $\mathrm{Si}^{34}$, carbon fiber ${ }^{14}$ and thin polyimide ${ }^{16,35}$ neural probes. The exceptionally small bending stiffness yields mechanical interaction with tissue in the range of cellular forces. For instance, the force to deflect the two sensor supporting arms by $10 \mu \mathrm{m}$ (scale of a cell) is estimated to be c.a. $10 \mathrm{nN}$, which is comparable to single cell migration force ${ }^{36}$ (Supplementary Methods).

Our fabrication exploits conventional planar 2D lithography with a sacrificial layer that is etched to yield the freestanding macroporous nanoelectronic probe (Fig. 1c). The overall design of the mesh probe (Fig. 1c; Supplementary Fig. S1 and Table S1) consists of longitudinal metal interconnects that are sandwiched between SU-8 polymer layers for passivation and transverse SU-8 polymer structural elements. In addition, transverse compressive strain elements are incorporated to generate positive transverse curvature to yield a cylindrical global probe structure, and local tensile strain elements in the supporting arms of each sensor device to produce negative curvature bending the devices away from the surface of the cylinder. All key materials and feature sizes of the macroporous nanoelectronic probe are summarized in Table S1. Facile I/O between the probe and measurement electronics is achieved by bonding a PCB connector to the remaining portion of the substrate attached to the freestanding macroporous probe following fabrication (Fig. 1d; Supplementary Fig. S2a).

The macroporous probes were fabricated on standard silicon wafers with a nickel release layer using photolithography for multi-layer patterning (Supplementary Methods; Supplementary Fig. S2). Low-to-high resolution optical images of a representative probe structure prior to 
etching the nickel release layer (Supplementary Fig. S2) highlight all key probe features. (1) The entire probe structure bonded to the PCB interface connector (Supplementary Fig. S2a). (2) The portion of the probe supported on the Ni layer and the silicon wafer that remains bonded to the PCB board (Supplementary Fig. S2b). (3) The lower part of the probe that will be released from the substrate (Supplementary Fig. S2c). The image also shows the overall locations of 19 sensor elements in this probe design. (4) Images of several addressable sensor elements, including one specific NW detector (Supplementary Figs. S2d, e).

Significantly, optical images of the probe structure following removal of the sacrificial nickel layer and underlying wafer (see Methods; Supplementary Methods), and immersion of the freestanding portion in aqueous buffer (Figs. 1e-i) highlight key features of our design. First, lower-resolution images (Fig. 1e) show that the transverse compressive strain elements produce self-organization of the probe into a global cylindrical shape as designed. This cylindrical geometry distributes the electronic sensor elements around the probe surface. Second, higher resolution images (Figs. 1f,g) demonstrate that the local tensile strain introduced in the supporting arms of each sensor element bend these arms outward such that each of the sensor elements is ca. $100 \mu \mathrm{m}$ away from the cylindrical probe surface. The average leakage impedance of the SU-8 encapsulated metal interconnect components (Supplementary Fig. S3) was $>10$ G $\Omega$ at relevant frequencies, which demonstrates the effectiveness of the SU-8 passivation used in the probes. For sensor elements, we have incorporated either silicon nanowire field effect transistors (FETs, Fig. 1h) or micrometer scale platinum metal electrodes (Fig. 1i) into our probes during fabrication (see Methods; Supplementary Methods). Characterization of device performance and neural recording with these different sensor elements are discussed below. 
The high flexibility of our macroporous probes precludes direct insertion ${ }^{14,20}$ into neural tissue, and invasive surgery, which could allow for placement of macroporous probes in specific brain regions, would largely eliminate many potential advantages of our design. To overcome this conundrum, we exploited the combination of built-in strain and liquid surface tension as probes are moved from liquid to air as shown schematically in Figure 2a and Supplementary Fig. S4. Specifically, upon withdrawing probes from liquid to air, liquid surface tension increases the global curvature to yield straight cylinders with diameters of ca. $100-200 \mu \mathrm{m}$ (Figs. 2b and Supplementary Mov. 1). In addition, surface tension causes the supporting arms of the sensors to be 'pulled back' to yield a smooth probe surface upon moving from liquid to air. Notably, the supporting arms return to the outward bent position with the sensors away from the probe surface when the probe is returned to an aqueous environment (Fig. 2b and Supplementary Mov. 2). We recently reported a syringe injection approach $2 \mathrm{D}$ mesh structures, although in this case all $\mathrm{I} / \mathrm{O}$ connections must be introduced after injection.

This cylindrical probe structure has mechanical advantages compared to a flat structure, and while it remains too soft to penetrate brain tissue as removed from solution, rapid freezing in liquid nitrogen (Fig. 2c) provides sufficient rigidity to allow for controlled insertion into hydrogel that has mechanical properties similar to dense neural tissue ${ }^{37}$. In particular, rapid insertion of the frozen probe into $0.5 \%$ agarose gel (Supplementary Fig. S5) yields an extended configuration $>2 \mathrm{~mm}$ into the hydrogel, while an unfrozen probe would simply be deformed at gel surface without penetration. To determine whether freezing could be a reliable insertion and measurement strategy, we also studied the electrical performance of fully assembled probes versus repeated liquid nitrogen freeze to room-temperature aqueous solution cycles. Significantly, characterization of a nanowire FET based probe following 150 freeze/thaw cycles (Fig. 2d) 
showed that 12 out of $14(86 \%)$ FET sensors on the probe remained connected, and that 14/14 devices remained active up to 21 cycles. In addition, the device sensitivities showed $<14 \%$ change on average after the 150 cycles, thus confirming the reliability of this approach. The observed robustness of the FET sensors is particularly encouraging because each device requires continuity of two interconnect lines versus a single line for metal electrode sensors.

We have implanted the macroporous nanoelectronic probes in rodents. In a typical implantation procedure (Fig. 3a), the frozen macroporous probe is stereotaxically positioned and rapidly inserted to a specific region of the brain of an anesthetized rodent (Methods). The positioning and inserting processes are kept within ca. 10 and 1 second, respectively, to ensure successful insertion (Supplementary Fig. S4). Images recorded post-insertion (Supplementary Fig. S6a) highlight the high flexibility of our macroporous probe outside the brain, which allows for positioning without moving the implanted portion within the tissue. In addition, a representative bright-field microscopy image of a post-insertion fixed tissue sample sectioned along the longitudinal axis of the probe (Supplementary Fig. S6b) verifies an extended linear structure within the brain tissue.

We have exploited the capability to target specific brain regions via stereotaxic insertion of the frozen macroporous nanoelectronic probes to test their in vivo recording capabilities in rodents, where all of the reported measurements are acute and performed within $0.5-2 \mathrm{~h}$ post implantation. First, a probe was implanted in the barrel cortex area of the rat brain since it represents a somatosensory cortex region with well-defined mapping between cortical columns and facial whiskers ${ }^{38}$. Signals recorded from four nanowire FET sensors showed strong signals in element-2 $(6.3 \pm 0.4 \mathrm{mV})$ corresponding to separate stimulations applied to the whisker $\mathrm{C} 1$ on the contralateral side of the implantation site (Fig. 3b), which was identified as the corresponding 
whisker by testing all whiskers (Supplementary Fig. S5). In contrast, the neighboring FET sensor element-3 recorded a similar but much weaker signal pattern, while elements 1 and 4, which are ca. 200 and $250 \mu \mathrm{m}$, respectively, from element-2, yielded no observable response.

Second, acute recording experiments made in the somatosensory cortex of an anesthetized rat (Fig. 3c) demonstrate the capability for larger-scale multiplexed recording with the macroporous nanoelectronic probes. Specifically, we recorded signals $(3.4 \pm 0.3 \mathrm{mV})$ simultaneously from 13 nanowire FET sensors on a single probe. The relatively large signal amplitude compared with that recorded by metal electrode sensors (typically $<0.5 \mathrm{mV}$ ) is attributed to the active sensing nature of the FET sensors, which do not suffer from signal loss by shunt pathways ${ }^{39}$. The dominant modulation frequency, $1-4 \mathrm{~Hz}$, is characteristic of $\delta$-wave local field potentials (LFPs) in anesthetized rats ${ }^{40}$. A spatial map of the recorded LFP is plotted in Supplementary Fig. S5a. The similarity and coherence between channels is consistent with the fact that the LFPs spread beyond ${ }^{41}$ the dimension of macroporous probe recording region, ca. $100 \mu \mathrm{m}$ laterally and $1 \mathrm{~mm}$ vertically. Multiplexed recording experiments were conducted more than ten times using nanowire FET sensor probes, and all experiments resulted in $>80 \%$ active sensor yield and similar recording performance in terms of potential shapes and amplitude. In addition, probes with platinum electrode sensors implanted in the somatosensory cortex region of a mouse brain (Fig. 3d) exhibited sharp millisecond spikes. Standard data processing and spikesorting (Methods, Fig. 3e) yielded a uniform potential waveform with average duration of $1.8 \mathrm{~ms}$ and peak-to-peak amplitude of $172 \mu \mathrm{V}$ characteristic of single-unit action potentials. The high signal to noise ratio $(>7)$ of the single-unit recording suggests a close proximity between the sensor and the firing neurons ${ }^{42}$, and thus provides at least comparable brain activity recordings as 
conventional probes ${ }^{13}$ but with the potential of being chronically much more biocompatible as discussed below.

The chronic response of neural tissue to our ultra-flexible macroporous probes have been addressed through histology studies carried out five weeks post implantation. A schematic of a macroporous probe inserted into the somatosensory cortex (Fig. 4a) highlights the perpendicular orientation (with respect to the implanted probe) that the tissue was sectioned at different times post-implantation. In general, slices were prepared after fixing the brain tissue using standard procedures (Methods) without removing the ultra-flexible macroporous nanoelectronic probes. Comparison of bright-field optical images recorded from similar acute (Fig. 4b, left) and chronic 5-week post implantation (Fig. 4b, right) tissue slices highlight several key points. First, the acute slice exhibits a tissue void within the interior of the roughly hollow cylindrical probe structure, which is consistent with ablation or displacement of tissue during implantation of the frozen probe. Second, images from the tissue 5-week post implantation shows no void, thus indicating that cells and/or neural projections interpenetrate through the macroporous probe over time to fill the acute void. In addition, analysis of chronic images (dashed white box, Fig. S8a) shows that at least some of the sensor arms can achieve the designed bend-out geometry postimplantation. This feature can facilitate positioning sensor devices away from any residual tissue damage resulting from implantation.

To evaluate more critically the chronic response of our macroporous nanoelectronic probes we used immunochemical staining ${ }^{14}$ of cross-section slices containing our probes. Confocal microscopy images of a region including a macroporous probe (Fig. 4c) show a normal growth density of neuron cell bodies (NeuN) in close proximity, $<50 \mu \mathrm{m}$, to the probe components (Supplementary Fig. S8d), although the soma density inside the probe cylinder is 
lower than outside. The close proximity of neuron cell bodies contrasts typical chronic observations reported for other types of neural probes ${ }^{4,19,25,43}$, which we discuss further below. In addition, glial fibrillary acidic protein (GFAP) expression is somewhat elevated at the center of the probe, but not at the outer edge of the probe or surrounding region. Indeed, the spatial dependence of the GFAP signal encompassing this outer probe edge is similar to that in the control sample (Fig 4d) prepared at the same time from the contralateral hemisphere without an implanted probe. These data suggest that the slightly elevated GFAP expression inside the probe cylinder is due to the acute tissue damage during implantation, and importantly, show that our ultra-flexible macroporous probes do not elicit chronic immune response post-implantation.

To further characterize the robustness of these results and the details of the chronic distribution of neurons about the macroporous probe we have stained tissue slices with $\beta$ tubulin-III, which can label both soma and neurites, from independent implantation experiments (Figs. 4e, f; Supplementary Fig. S8). Significantly, confocal microscopy images recorded 5 weeks post-implantation show no significant drop in $\beta$-tubulin-III expression inside (9.8 a.u., average) versus outside (10.2 a.u., average) the probe. These results suggest that neural projections, unlike somas, have sufficient mobility to interpenetrate and partially fill the central void produced during initial implantation. Moreover, analyses of the fluorescent intensity in the region immediately outside the probe (Fig. 4f; Supplementary Fig. S8c) show no sign of suppressed neuron growth immediately adjacent to the probe structural elements, and indeed, these data indicate that the neuronal fluorescence signal within $10 \mu \mathrm{m}$ of the probe components (20-50, average) is more than $2-5 x$ the value for neurons averaged over the entire image. ). In addition, measurements made from tissue/probe slice $150 \mu \mathrm{m}$ deeper in the brain than shown in Fig. 4c (Supplementary Fig. S8e) and from an independent probe implant that partially collapsed 
during slow insertion (Supplementary Fig. S8f) exhibited similar results, and thus indicate that the macroporous probe structure is attractive to neurons (e.g., neurophilic) and does not illicit the usual immune response. Similar results are observed in studies of syringe injected mesh electronics $^{32}$, although conventional micro-wire ${ }^{4,25}$ and silicon ${ }^{19,43}$ neural probes as well as ultrasmall but rigid $^{14}$ and flexible two-dimensional polymer probes ${ }^{16,} 17$ show enhanced GFAP/astrocyte proliferation and a reduction of neuron density near these probe surface. Since chronic failure of conventional brain probes involves neuronal loss and the encapsulation of nonneuronal cells such as astrocytes up to several hundred micrometers from the probe surface ${ }^{4,43}$, our results suggest substantial benefits of the macroporous nanoelectronic probes for future chronic recording studies.

In summary, our 3D macroporous nanoelectronic probes, which feature ultra-flexibility comparable to neural tissue and open structures with sub-cellular feature sizes allowing for neuron interpenetration, represent a new strategy to merge 3D nanoelectronic devices with the neural circuits in the brain. We have shown that the ultra-flexible macroporous probes can be stereotaxically-implanted in a frozen state into rodent brains with minimal surgical and acute tissue damage, and demonstrated the capability of recording multiplexed LFPs and single-unit action potentials from the somatosensory cortex. Significantly, chronic histology studies revealed unique characteristics, including a filling-in of neural tissue through the macroporous network and attractive neuron-probe interactions, which contrast results from other solid and more rigid probe designs ${ }^{19,20}$, and are consistent with a unique long-term stability and biocompatibility of the probe-tissue interface. While it will be important in future studies to develop further these probes, for example by extending the chronic histology studies to shorter and longer times and increasing the number of sensor elements available for multiplexed recording and/or introducing 
stimulation capabilities, we believe the present chronic histology and acute recording studies already show unique advantages of our ultra-flexible 3D macroporous electronic probes and point to the importance of exploring the stability of chronic neural activity mapping and implants for next generation brain-machine interfaces in the near future.

\section{References}

1. Alivisatos, A. P., et al. Neuroscience. The brain activity map. Science 339, 1284-1285 (2013).

2. Birmingham, K., et al. Bioelectronic medicines: a research roadmap. Nat. Rev. Drug Discov. 13, 399-400 (2014).

3. Shen, H. Neurotechnology: BRAIN storm. Nature 503, 26-28 (2013).

4. Polikov, V. S., Tresco, P. A. \& Reichert, W. M. Response of brain tissue to chronically implanted neural electrodes. J. Neurosci. Methods 148, 1-18 (2005).

5. Olsen, S. R., Bortone, D. S., Adesnik, H. \& Scanziani, M. Gain control by layer six in cortical circuits of vision. Nature 483, 47-52 (2012).

6. Mizuseki, K., Diba, K., Pastalkova, E. \& Buzsaki, G. Hippocampal CA1 pyramidal cells form functionally distinct sublayers. Nat. Neurosci. 14, 1174-1181 (2011).

7. Hochberg, L. R., et al. Reach and grasp by people with tetraplegia using a neurally controlled robotic arm. Nature 485, 372-375 (2012).

8. Nicolelis, M. A. Actions from thoughts. Nature 409, 403-407 (2001).

9. Taylor, D. M., Tillery, S. I. \& Schwartz, A. B. Direct cortical control of 3D neuroprosthetic devices. Science 296, 1829-1832 (2002).

10. Perlmutter, J. S. \& Mink, J. W. Deep brain stimulation. Annu. Rev. Neurosci. 29, 229257 (2006).

11. Seymour, J. P. \& Kipke, D. R. Neural probe design for reduced tissue encapsulation in CNS. Biomaterials 28, 3594-3607 (2007). 
12. Kozai, T. D. \& Kipke, D. R. Insertion shuttle with carboxyl terminated self-assembled monolayer coatings for implanting flexible polymer neural probes in the brain. $J$. Neurosci. Methods 184, 199-205 (2009).

13. HajjHassan, M., Chodavarapu, V. \& Musallam, S. NeuroMEMS: Neural Probe Microtechnologies. Sensors 8, 6704-6726 (2008).

14. Kozai, T. D., et al. Ultrasmall implantable composite microelectrodes with bioactive surfaces for chronic neural interfaces. Nat. Mater. 11, 1065-1073 (2012).

15. Seymour, J. P. \& Kipke, D. R. Fabrication of polymer neural probes with sub-cellular features for reduced tissue encapsulation. Conf. Proc. IEEE Eng. Med. Biol. Soc. 1, 4606-4609 (2006).

16. Kim, T. I., et al. Injectable, cellular-scale optoelectronics with applications for wireless optogenetics. Science 340, 211-216 (2013).

17. Sohal, H. S., et al. The sinusoidal probe: a new approach to improve electrode longevity. Front. Neuroeng 10, 1-14 (2014).

18. Nicolelis, M. A., et al. Chronic, multisite, multielectrode recordings in macaque monkeys. Proc. Natl. Acad. Sci. U. S. A. 100, 11041-11046 (2003).

19. Rousche, P. J. \& Normann, R. A. Chronic recording capability of the Utah Intracortical Electrode Array in cat sensory cortex. J. Neurosci. Methods 82, 1-15 (1998).

20. Kipke, D. R., Vetter, R. J., Williams, J. C. \& Hetke, J. F. Silicon-substrate intracortical microelectrode arrays for long-term recording of neuronal spike activity in cerebral cortex. IEEE Trans. Neural Syst. Rehabil. Eng. 11, 151-155 (2003).

21. Miller, K., Chinzei, K., Orssengo, G. \& Bednarz, P. Mechanical properties of brain tissue in-vivo: experiment and computer simulation. J. Biomech. 33, 1369-1376 (2000).

22. Perge, J. A., et al. Intra-day signal instabilities affect decoding performance in an intracortical neural interface system. J. Neural Eng. 10, 036004 (2013).

23. Biran, R., Martin, D. C. \& Tresco, P. A. The brain tissue response to implanted silicon microelectrode arrays is increased when the device is tethered to the skull. J. Biomed. Mater. Res. A 82, 169-178 (2007).

24. Dickey, A. S., Suminski, A., Amit, Y. \& Hatsopoulos, N. G. Single-unit stability using chronically implanted multielectrode arrays. J. Neurophysiol. 102, 1331-1339 (2009). 
25. Jackson, A. \& Fetz, E. E. Compact movable microwire array for long-term chronic unit recording in cerebral cortex of primates. J. Neurophysiol. 98, 3109-3118 (2007).

26. Tee, B. C., Wang, C., Allen, R. \& Bao, Z. An electrically and mechanically self-healing composite with pressure- and flexion-sensitive properties for electronic skin applications. Nature nanotechnology 7, 825-832 (2012).

27. Mannoor, M. S., et al. 3D printed bionic ears. Nano Lett. 13, 2634-2639 (2013).

28. Jeong, J. W., et al. Soft Materials in Neuroengineering for Hard Problems in Neuroscience. Neuron 86, 175-186 (2015).

29. Spira, M. E. \& Hai, A. Multi-electrode array technologies for neuroscience and cardiology. Nature nanotechnology 8, 83-94 (2013).

30. Kruskal, P. B., Jiang, Z., Gao, T. \& Lieber, C. M. Beyond the patch clamp: nanotechnologies for intracellular recording. Neuron 86, 21-24 (2015).

31. Tian, B., et al. Macroporous nanowire nanoelectronic scaffolds for synthetic tissues. Nat. Mater. 11, 986-994 (2012).

32. Liu, J., et al. Syringe-injectable electronics. Nature nanotechnology 10, 629-636 (2015).

33. Liu, J., et al. Multifunctional three-dimensional macroporous nanoelectronic networks for smart materials. Proc. Natl. Acad. Sci. U. S. A. 110, 6694-6699 (2013).

34. Lee, H., Bellamkonda, R. V., Sun, W. \& Levenston, M. E. Biomechanical analysis of silicon microelectrode-induced strain in the brain. J. Neural Eng. 2, 81-89 (2005).

35. Rousche, P. J., et al. Flexible polyimide-based intracortical electrode arrays with bioactive capability. IEEE Trans. Biomed. Eng. 48, 361-371 (2001).

36. du Roure, O., et al. Force mapping in epithelial cell migration. Proc. Natl. Acad. Sci. U. S. A. 102, 2390-2395 (2005).

37. Sharp, A. A., Ortega, A. M., Restrepo, D., Curran-Everett, D. \& Gall, K. In vivo penetration mechanics and mechanical properties of mouse brain tissue at micrometer scales. IEEE Trans. Biomed. Eng. 56, 45-53 (2009).

38. Petersen, C. C. The functional organization of the barrel cortex. Neuron 56, 339-355 (2007).

39. Boulton, A. A., Baker, G. B. \& Vanderwolf, C. H. Neurophysiological techniques. Humana Press: Clifton, N.J., 1990. 
40. Sheeba, J. H., Stefanovska, A. \& McClintock, P. V. Neuronal synchrony during anesthesia: a thalamocortical model. Biophys. J. 95, 2722-2727 (2008).

41. Kajikawa, Y. \& Schroeder, C. E. How Local Is the Local Field Potential? Neuron 72, 847-858 (2011).

42. Stratton, P., et al. Action potential waveform variability limits multi-unit separation in freely behaving rats. PloS one 7, e38482 (2012).

43. Biran, R., Martin, D. C. \& Tresco, P. A. Neuronal cell loss accompanies the brain tissue response to chronically implanted silicon microelectrode arrays. Exp. Neurol. 195, 115-126 (2005).

\section{Methods}

\section{Macroporous nanoelectronic brain probe preparation}

Macroporous nanoelectronic probes were fabricated using methods described previously ${ }^{18,27}$ (see Supplementary Methods for details). In brief, the 2D probe structure was fabricated using photolithography on a nickel metal release layer deposited on a silicon substrate (600nm $\mathrm{SiO}_{2}$ or $100 \mathrm{SiO}_{2} / 200 \mathrm{Si}_{3} \mathrm{~N}_{4}, n$-type $0.005 \mathrm{~V} \cdot \mathrm{cm}$, Nova Electronic Materials, Flower Mound, TX). After fabrication, a custom-designed PCB connector was mounted on the silicon substrate next to the contact region of the probe. Connections from the contact pads to the connector were made by wire bonding. Subsequently, the relief region of the probe was soaked in nickel etchant (TFB, Transene Company Inc., Danvers, MA) for $60-120$ min at $25{ }^{\circ} \mathrm{C}$ to release the free standing portion of the probe, while the remainder of the probe is attached to the substrate. The substrate was trimmed to the size of the contact region prior to implantation.

\section{Stereotaxic surgery and probe implantation}

The macroporous nanoelectronic brain probe implantation was carried following a standard rodent stereotaxic surgery protocol. Briefly, the rat or mouse was anesthetized with 
intraperitoneally administered ketamine/xylazine (Patterson Veterinary Supply Inc., Chicago, IL), and held in a stereotaxic frame (Lab Standard Stereotaxic Instrument, Stoelting Co., Wood Dale, IL). A hole in the cranium $(6 \mathrm{~mm} \times 6 \mathrm{~mm})$ was milled with a dental drill (Micromotor with On/Off Pedal 110/220, Grobet USA, Carlstadt, NJ) above the somatosensory cortex area. The dura was incised and resected. The probe was then implanted stereotaxically in the frozen state. Acute recording was performed $0.5-2$ hours after the implantation. For chronic experiments, the surgical closure and probe connector fixture on the skull were achieved with C\&B-METABOND (Cement System, Parkell, Inc., Edgewood, NY). Anti-inflammatory and anti-bacterial ointment was swabbed onto the skin after surgery. A $0.3 \mathrm{~mL}$ intraperitoneal injection of Buprenex (Patterson Veterinary Supply Inc. Chicago, IL, diluted with $0.5 \mathrm{ml}$ of PBS) for $0.1 \mathrm{mg} / \mathrm{kg}$ was administered to reduce post-operative pain. Animals were observed for 4 hours after surgery and hydrogel was provided for food and water, and heating pad at $37{ }^{\circ} \mathrm{C}$ for the remainder of post-operative care. All animal procedures conformed to US National Institutes of Health guidelines and were approved by Harvard University's Animal Care and Use Committee.

\section{Incubation and behavioral analysis}

Animals were cared every day for 3 days after the surgery and every other day after the first 3 days. Animals were administered $0.3 \mathrm{~mL}$ of Buprenex $(0.1 \mathrm{mg} / \mathrm{kg}$, diluted with $0.5 \mathrm{~mL} 1 \mathrm{X}$ PBS) every 12 hours for 3 days. Animals were also observed every other day for behavioral changes. The room was maintained at constant temperature on a 12-12 h light-dark cycle.

\section{Voltage sensing using the nanowire FET sensors and Pt electrodes in the macroporous nanoelectronic brain probe}


Nanowire device recording was carried out with a custom-built $100 \mathrm{mV}$ DC voltage source, and the current was amplified with a custom-built 16-channel current/voltage preamplifier with a typical gain setting of $10^{6} \mathrm{~A} / \mathrm{V}$. The signals were filtered with a $3 \mathrm{kHz}$ low pass filter (CyberAmp 380, Molecular Devices, Sunnyvale, CA), and digitized at a sampling rate of $20 \mathrm{kHz}$ (AxonDigi1440A, Molecular Devices, Sunnyvale, CA) and recorded using Clampex 10 software (Molecular Devices, Sunnyvale, CA). The measured conductance changes of nanowire devices were translated to potential according to a calibration curves measured after implantation $^{33}$. All nanowire devices were tested for functionality before recording. Voltage signals from Pt electrode sensors were recording using a 32-channel Intan RHD 2132 amplifier evaluation system (Intan Technologies LLC., Los Angeles, CA) with an Ag/AgCl electrode acting as the reference and counter electrode. The sampling rate was $20 \mathrm{kHz}$, and a $300-6000$ $\mathrm{Hz}$ band pass filter was applied for single unit recording. Spike-sorting was performed using Clampfit (Molecular Devices, Sunnyvale, CA). The impedance of the Pt electrodes was measured by the same equipment. The AC leakage impedance of the probe components was measured by a B1500A semiconductor parameter analyzer (Keysight, Santa Rosa, CA)

\section{Histology sample preparation}

After anesthesia (described above) was induced, the animal was perfused transcardially with PBS and then $40 \mathrm{~mL}$ 4\% paraformaldehyde (Sigma-Aldrich Corp., St. Louis, MO). The animal was decapitated and the brain was removed from the skull and set in $4 \%$ formaldehyde for 24 hours as post fixation. The brain was then transferred to incrementally increasing sucrose solutions (10-30\%) (Sigma-Aldrich Corp., St. Louis, MO) for cryoprotection and followed by sectioning into $10-150 \mu \mathrm{m}$ slices perpendicular or parallel to the probe using Leica CM1950 cryostat (Leica Microsystems Inc., Buffalo Grove, IL). The slices were pre-blocked and 
permeabilized $(0.2-0.25 \%$ Triton $\mathrm{X}-100$ and $1 \%$ bovine serum albumin (BSA) in PBS, (SigmaAldrich Corp., St. Louis, MO)) for 1 hour at room temperature. Next the slices were incubated with primary antibodies containing $0.2 \%$ triton and $3 \%$ serum overnight at $4{ }^{\circ} \mathrm{C}$, and then were incubated with the secondary antibodies with fluorophores overnight at $4{ }^{\circ} \mathrm{C}$. For counterstaining of cell nuclei, cells were incubated with $0.1-1 \mu \mathrm{g} / \mathrm{mL}$ Hoechst 34580 (Invitrogen, Carlsbad, CA) for $1 \mathrm{~min}$.

Reagents used for different cell types are as follows (all from AbCam, Cambridge, MA). Neuron: Rabbit polyclonal to NeuN and Rabbit polyclonal to $\beta$-Tubulin-III were used as primary antibodies. Goat anti-rabbit IgG AlexaFlour 488 was used as the second antibody. Astrocyte: Chicken polyclonal to GFAP and Goat anti-chicken IgG alexaFlour 647 were used as the primary and secondary antibodies respectively.

\section{Structural and fluorescent imaging}

Bright-field, dark-field and confocal fluorescent micrographs of samples were acquired on an Olympus BX61 microscope (Olympus America Inc., Center Valley, PA) or a Zeiss LSM 780 confocal microscope (Carl Zeiss Microscopy, Thornwood, NY). Confocal images were acquired using $405,473,559$ and $635 \mathrm{~nm}$ wavelength lasers as excitation and a spectrum detector collecting emission. ImageJ (ver. 1.45i, Wayne Rasband, National Institutes of Health, USA) or Zen (Carl Zeiss Microscopy, Thornwood, NY) was used for analysis of the image data.

\section{Acknowledgments}

We thank J. Tian for the help and discussions on animal surgeries. This study was supported by Air Force Office of Scientific Research and NSSEFF awards (C.M.L.). 


\section{Author Contributions}

C.X., J.L., and C.M.L. conceived the idea and designed the experiments. C.X., J.L., T.F, X.D., and W.Z. performed the experiments and analyses. C.X. and C.M.L. wrote the manuscript. All authors discussed the results, interpreted the findings and reviewed the manuscript.

\section{Competing financial interests}

The authors declare no competing financial interests.

\section{Figure Legends}

Figure 1. Macroporous nanoelectronic 3D neural probes. a, Schematic of the probe implanted in the brain. The macroporous and flexible probe, indicated by the yellow lines, is implanted in the brain and connected to the cranially mounted I/O connector. b, Schematic of the microscopic interface of the macroporous nanoelectronic brain probe with the neural circuit. The light blue lines represent the polymer-encapsulated metal interconnects and supporting elements. The orange lines represent arms that support and connect sensors. c, Schematic of the mechanism of probe geometry control by built-in strain after the removal of the sacrificial layer. Compressive strain elements, which are indicated by blue colored lines along the transverse direction, shape the probe into a cylindrical structure. Tensile strain elements, which are indicated by red color, cause the sensor supporting arms to bend outward from the probe surface. d, Schematic of an assembled macroporous probe with I/O connector. The front end of the probe, represented by light purple lines, is suspended in buffer and the back end, represented by orange pads, is attached to a carrier substrate and connected to an I/O connector. e, Photograph of a typical macroporous nanoelectronic brain probe suspended in buffer with a cylindrical shape. Its back end is attached to the carrier substrate (the dark piece in the back) at the top of the image. Scale bar: $500 \mu \mathrm{m}$. f, Micrograph of the sensor area of the probe outlined by the red dashed box 
in e. The self-organization of the probe geometry, including global scrolling and outward bent supporting arms are visible. Scale bar: $200 \mu \mathrm{m}$. g, Zoomed-in view of the outward bent supporting arm and sensor outlined by the yellow dashed box in $\mathbf{f}$. The black dashed box highlights the sensor element. Scale bar: $50 \mu \mathrm{m}$. h, Dark field micrograph of a typical nanowire FET voltage sensor at the end of the supporting arms. The arrow points a nanowire as the sensor unit. Scale bar: $5 \mu \mathrm{m}$. i, Bright field micrograph of two typical Pt electrode voltage sensors each with $4 \mu \mathrm{m} \times 20 \mu \mathrm{m}$ area. Scale bar: $5 \mu \mathrm{m}$.

Figure 2. Probe preparation and robustness. a, Schematic of the probe geometry-control before implantation. The global curvature of the probe increases and the supporting arms are flattened as the probe is pulled liquid to air. b, Four time-lapse images of the probe front end geometry while it was moving in and out of the buffer. The dashed line marks the buffer surface (air above; buffer below). Large black arrows indicate direction of motion in panels 2-4. Scale bar: $200 \mu \mathrm{m}$. c, Photograph of the probe in the 'frozen' state held in air by the carrier substrate. Scale bar: $500 \mu \mathrm{m}$. d, Sensor yield test following liquid nitrogen freezing and room-temperature thaw cycles. Left: Number of active nanowire FET sensors versus free/thaw cycle number. Right: Nanowire FET transconductance versus cycle number for 6 out of the 14 sensor elements.

Figure 3. Neural activity recording from rodent models. a, Photograph of a typical rodent stereotaxic surgery. A rat was held in a stereotaxic frame. A macroporous nanoelectronic probe was implanted into the brain through a cranial hole. The probe was attached to the carrier substrate for external electrical connections (Supplementary Methods). b, Acute LFP recording 
by nanowire FET sensors from the barrel cortex area. Left: Schematic of the correlation between the neural activity in barrel cortex and the rat whisker sensory behavior. Inset: schematic map of sub-areas in barrel cortex. The red circle indicates the targeted sub-area. Right: Traces from four neighboring sensors, where yellow areas mark stimulations applied to the whisker C1. Relative positions of the four sensors are marked in the schematic on the right. Scale bar: $200 \mu \mathrm{m}$. c, Acute multiplexed LFP recording from 13 nanowire FET sensors following probe insertion into the somatosensory cortex. Relative positions of the 13 sensors are marked in the schematic on the left. Scale bar: $200 \mu \mathrm{m}$. d, Top: Representative acute single unit recording from Pt electrode sensors. Bottom: zoomed-in view of 9 single unit events outlined in the top panel. e, Superimposed 94 single unit events from the recording in $\mathbf{d}$. The mean waveform of all traces is plotted in red.

Figure 4. Implanted macroporous nanoelectronic probe-tissue histology. a, Schematic of brain slice sample preparation. The dashed line indicates the slice direction perpendicular to the implanted macroporous probe, represented by light blue lines. b, Bright field images of the probe-tissue interface cross-section. The dark objects in the image are components of the probe. Left: bright field image of a $100 \mu \mathrm{m}$ thick acute slice. Scale bar: $100 \mu \mathrm{m}$. Right: Bright field image of a $20 \mu \mathrm{m}$ thick cross-section slice 5 weeks after implantation. The white dashed box highlights the area imaged and shown in Fig. 4e. Scale bar: $20 \mu \mathrm{m}$. c, Projection of 3D reconstructed confocal micrograph of immunochemically labeled cross-section slice in $\mathbf{b}$ right (5 weeks post implantation). The pseudo color coding is as follows. Blue: nucleus, Hoechst; green: NeuN, labeling neuron nuclei; white: SU-8; and red: GFAP, specifically labeling reactive astrocytes. The fluorescent intensity profiles of the red channel (astrocyte) along the long-axis of 
the two dashed box areas are plotted in the right panel. Scale bar: $20 \mu \mathrm{m}$. Similar data from a slice obtained ca. $150 \mu \mathrm{m}$ deeper in the brain on this same probe is shown in Supplementary Fig. S8e. d, Immunochemical staining image of the control sample for $\mathbf{c}$ obtained from the contralateral hemisphere of the same mouse. The fluorescent intensity profiles of GFAP along the long-axis of the two dashed box areas are plotted in the right panel. Scale bar: $20 \mu \mathrm{m}$. e, Projection of 3D reconstructed confocal micrograph of immunochemically labeled cross-section slice in b. Right. The pseudo color-coding is as follows. Blue: nucleus, Hoechst; green: $\beta$ tubulin-III; orange: SU-8, and red: GFAP. Scale bar: $10 \mu \mathrm{m}$. f, Green channel ( $\beta$-tubulin-III) fluorescence intensity plotted along the outer curved portion of the probe outlined area in the dashed box in $\mathbf{e}$, from left to right. Orange bars indicate the positions of the mesh components. The blue dashed line indicates the average of the $\beta$-tubulin-III fluorescence intensity for the entire imaged area in $\mathbf{e}$. All tissue slices were prepared post implantation into the somatosensory cortex region of mice (Methods) as shown schematically in a. 


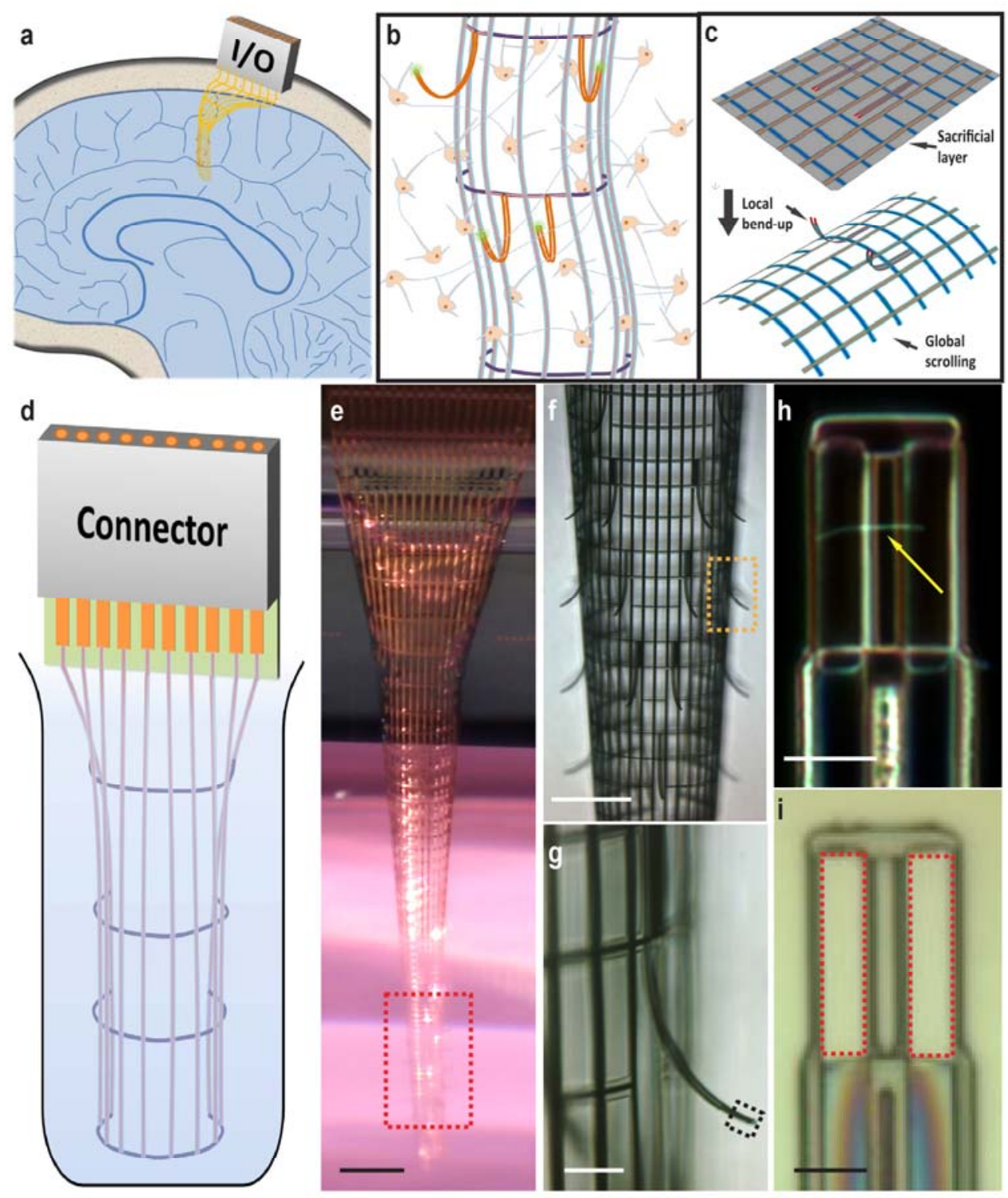

Figure 1 


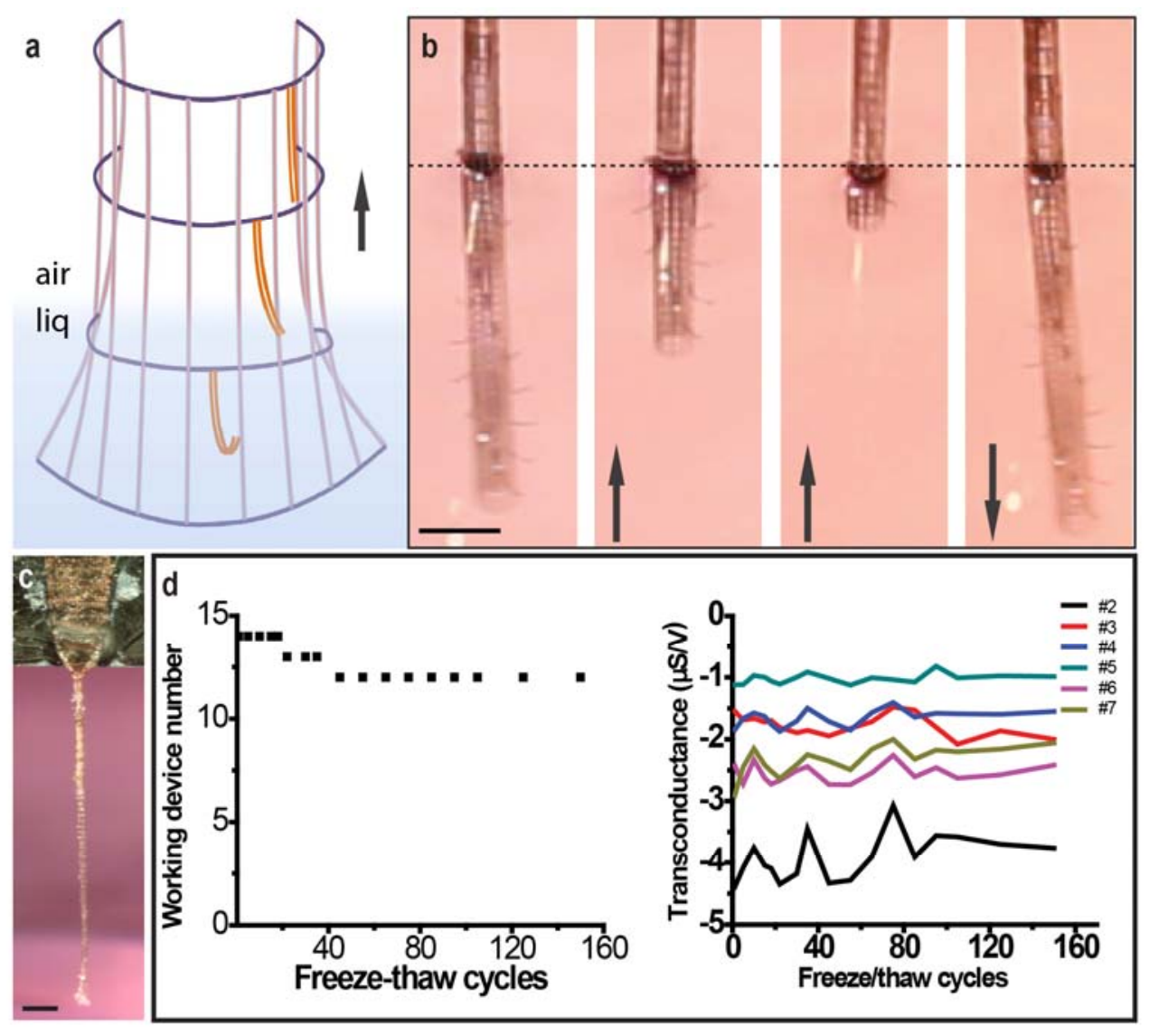

Figure 2 


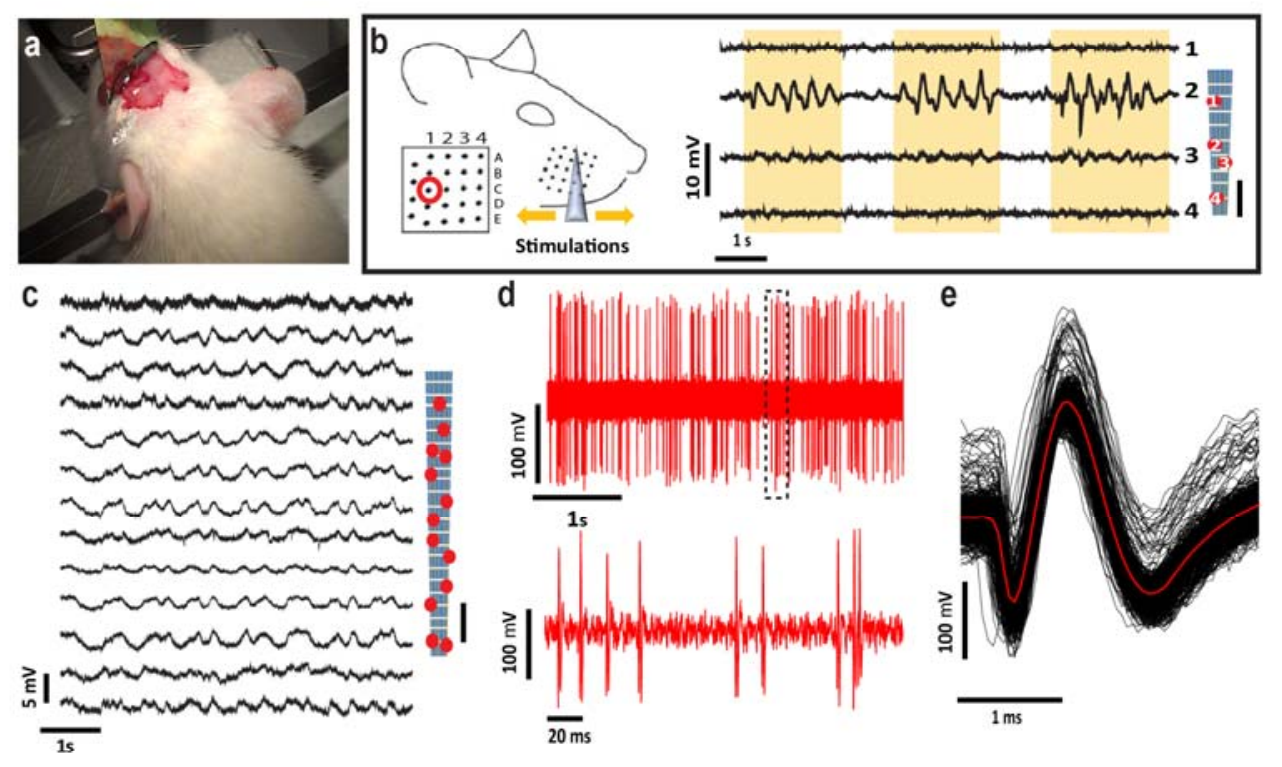

Figure 3 

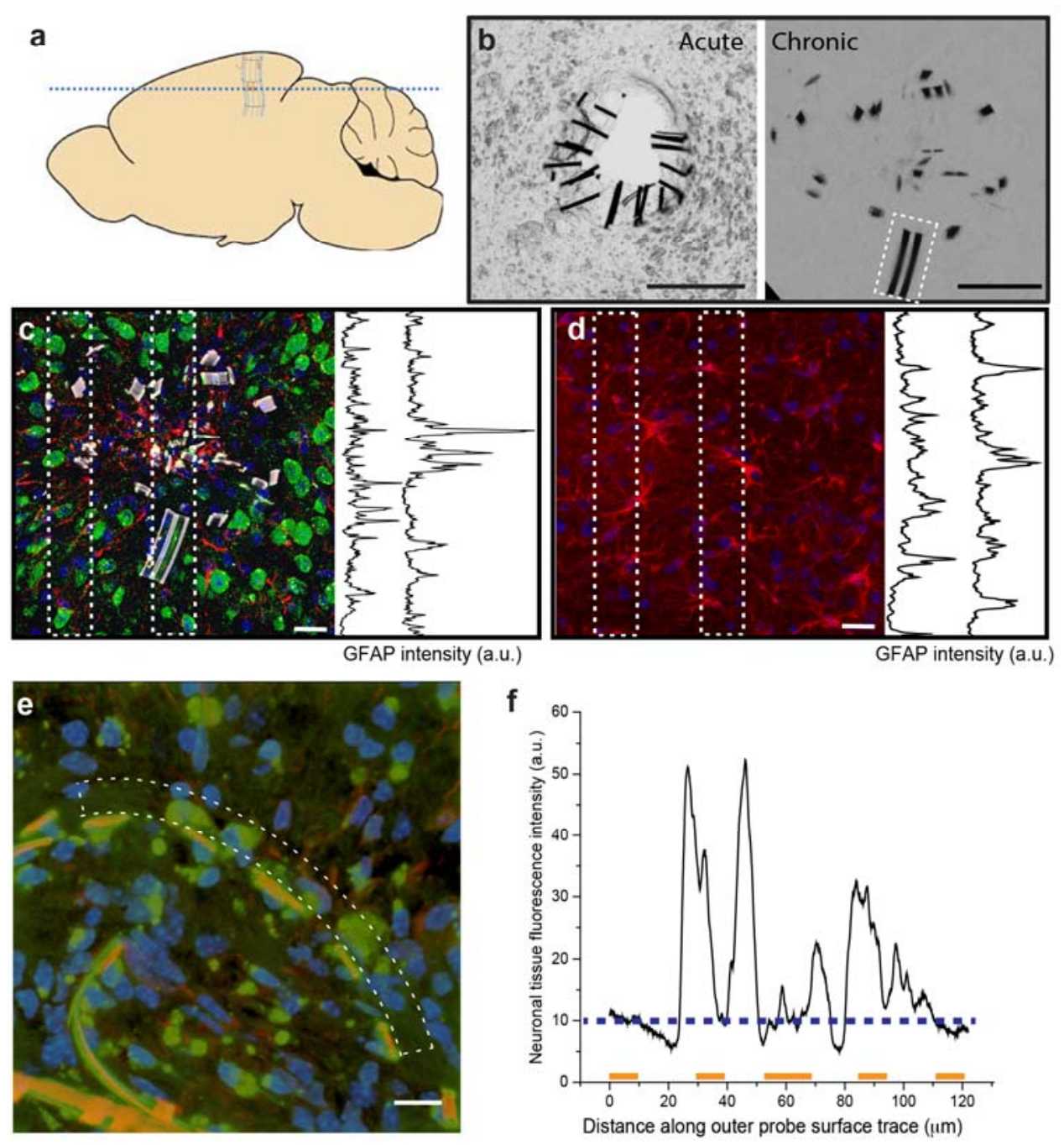

Figure 4 
Supplementary Information for:

\section{Three-dimensional macroporous nanoelectronic networks as minimally-invasive brain probes}

Chong Xie, Jia Liu, Tian-Ming Fu, Xiaochuan Dai, Wei Zhou and Charles M. Lieber

This file includes:

Supplementary Methods

Figures S1-S8 


\section{Supplementary Methods}

\section{Nanowire Synthesis.}

Uniform $30 \mathrm{~nm} p$-type single crystal silicon nanowires were synthesized using our reported gold nanocluster-catalyzed vapor-liquid-solid methodology ${ }^{\mathrm{S} 1}$. In a typical synthesis, the total pressure was 40 torr and the flow rates of $\mathrm{SiH}_{4}$, diborane $\left(\mathrm{B}_{2} \mathrm{H}_{6}, 100\right.$ p.p.m. in $\left.\mathrm{H}_{2}\right)$, and hydrogen $\left(\mathrm{H}_{2}\right.$, Semiconductor Grade), were 2, 2.5 and 60 standard cubic centimetres per minute (SCCM), respectively. The silicon-boron feed-in ratio was 4000:1, and the total nanowire growth time was $30 \mathrm{~min}$.

\section{Macroporous nanoelectronic brain probe fabrication}

The macroporous nanoelectronic brain probes were fabricated with key steps as follows: $(i)$ photolithography and thermal deposition were used to pattern a $100 \mathrm{~nm}$ nickel sacrificial layer, where the nickel served as the final relief layer for the free-standing probe. (ii) Photolithography and thermal deposition were used to define the $\mathrm{Cr} / \mathrm{Pd} / \mathrm{Cr}(10-20 / 80 / 1.5 \mathrm{~nm})$ non-symmetric metal

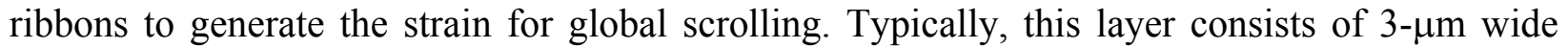
parallel ribbons. (iii) A 300-500 nm layer of SU-8 photoresist was defined by photolithography as the bottom SU-8 passivation layer. Typically, this layer consists of $7-\mu \mathrm{m}$ wide parallel ribbons. (iv) Either nanowire FETs or Pt electrodes were patterned as the voltage sensors (details in the following section). (v) Photolithography and thermal deposition were used to define the $\mathrm{Cr} / \mathrm{Pd} / \mathrm{Cr}(1.5 / 80 / 50-80 \mathrm{~nm})$ double metal ribbons to generate strain for local bend-out from the global cylindrical structure. Typically, this layer consists of 3- $\mu \mathrm{m}$ wide metal interconnect lines. (vi) Photolithography and thermal deposition were used to define the non-strained metal contacts, $\mathrm{Cr} / \mathrm{Au} / \mathrm{Cr}(1.5 / 100 / 1.5 \mathrm{~nm})$, to address each sensor and form interconnections to the input/output pads, which are patterned outside the Ni sacrificial layer. This layer usually consists 
of 5- $\mu \mathrm{m}$ wide metal interconnect lines. (vii) Another 300-500 nm thick layer of SU-8 photoresist was defined by photolithography as the top SU-8 passivation. This layer typically has the same pattern as the bottom SU-8 passivation layer in (iii).

It should be noted that $\mathrm{Pd}$ is a toxic metal and may cause side effect if exposed. In this work the $\mathrm{Pd}$ is well protected by the SU8 encapsulation. However, in future long-term applications, other metals, such as $\mathrm{Pt}$ or $\mathrm{Ti}$, which are known to make good contact with $\mathrm{Si}$

nanowire $^{\mathrm{S} 2}$ and can provide controllable strain $^{\mathrm{S} 3}$, can be used to replace $\mathrm{Pd}$ to eliminate possible toxicity.

\section{Nanowire FET sensor patterning.}

(a) A 300 to $400 \mathrm{~nm}$ layer of SU-8 photoresist was deposited on the fabrication substrate, prebaked $\left(65^{\circ} \mathrm{C} / 2 \mathrm{~min} ; 95^{\circ} \mathrm{C} / 4 \mathrm{~min}\right)$, and then (b) silicon nanowires were aligned on the SU-8 layer by contact printing as described previously ${ }^{\mathrm{S}}$. (c) Photolithography was used to define the nanowire device regions, and after post-baking $\left(65^{\circ} \mathrm{C} / 2 \mathrm{~min} ; 95^{\circ} \mathrm{C} / 2 \mathrm{~min}\right)$, the pattern was developed by SU-8 Developer washed with isopropanol (2 times, $30 \mathrm{~s}$ per wash) to remove nanowires outside of the device regions. (d) The new SU-8 pattern was cured at $180{ }^{\circ} \mathrm{C} / 20 \mathrm{~min}$. (e) Nanowire device element contacts were defined by photolithography and $\mathrm{Cr} / \mathrm{Pd} / \mathrm{Cr}(1.5 / 50-$ $80 / 1.5 \mathrm{~nm})$ metallization.

\section{Metal electrode sensor patterning.}

$\mathrm{Cr} / \mathrm{Pt}(1.5 / 100 \mathrm{~nm})$ electrodes are patterned by photolithography. The electrode size used in this work was $4 \mu \mathrm{m} \times 20 \mu \mathrm{m}$, with typical impedance of $600 \pm 20 \mathrm{kohm}$ at $1 \mathrm{kHz}$.

\section{Calculation of the bending stiffness for different neural probes.}


The mechanical characteristics of the three-layer longitudinal ribbons make the dominant contribution to the probe-tissue interface. The bending stiffness of a single ribbon, $K_{I R}$, can be estimated as ${ }^{\mathrm{S} 5}$

$$
K_{1 R}=E_{s}\left(\frac{h^{3} w_{1}}{12}-\frac{h_{m}^{3} w_{m}}{12}\right)+E_{m} \frac{h_{m}^{3} w_{m}}{12}
$$

where $E_{s}$ is young's modulus of SU-8, $E_{m}$ is young's modulus of gold, $h$ is the total thickness of ribbon, $h_{m}$ is the thickness of metal, $w_{l}$ is the total width of ribbon and $w_{m}$ is the width of metal. When $E_{s}=2 \mathrm{GPa}, E_{m}=79 \mathrm{GPa}, h=800 \mathrm{~nm}, h_{m}=100 \mathrm{~nm}, w_{l}=7 \mu \mathrm{m}$, and $w_{m}=5 \mu \mathrm{m}, K_{l R}=$ $0.64 \times 10^{-15} \mathrm{~N} \cdot \mathrm{m}^{2}$.

The bending stiffness of another representative component of the probe, the sensor device support arm, $K_{I S}$, is calculated similarly taking $w_{l}=6 \mu \mathrm{m}, w_{m}=4 \mu \mathrm{m} . K_{I S}=0.54 \times 10^{-15} \mathrm{~N} \cdot \mathrm{m}^{2}$.

The bending stiffness of standard silicon probes or planar thin film probes, $K_{2}$, can be estimated as $^{\mathrm{S} 5}$

$$
K_{2}=E \frac{w h^{3}}{12}
$$

where $E$ is the young's modulus of the probe material, $h$ is the thickness of the probe, and $w$ is the width of the probe. When $E_{\text {silicon }}=165 \mathrm{GPa}, h_{\text {silicon }}=15 \mu \mathrm{m}$, and $w=100 \mu \mathrm{m}$, the bending stiffness of a typical silicon probe is $K_{2 s}=4.6 \times 10^{-8} \mathrm{~N} \cdot \mathrm{m}^{2}$. When $E_{\text {polyimide }}=2 \mathrm{GPa}, \mathrm{h}_{\text {polyimide }}=10$ $\mu \mathrm{m}$, and $w=100 \mu \mathrm{m}$, the bending stiffness of a typical polyimide probe is $\mathrm{K}_{2 \mathrm{P}}=0.16 \times 10^{-10}$ $\mathrm{N} \cdot \mathrm{m}^{2}$.

The bending stiffness of ultrasmall carbon electrodes, $K_{3}$, can be estimated as ${ }^{\mathrm{S} 5}$

$$
K_{3}=E_{\text {carbon }} \frac{\pi d^{4}}{64}
$$

Where $E_{\text {carbon }}$ is the young's modulus of carbon fiber, $d$ is the diameter of carbon fiber probe. When $E_{\text {carbon }}=234 \mathrm{GPa}, d=7 \mu \mathrm{m}, K_{3}=2.73 \times 10^{-10} \mathrm{nN} \cdot \mathrm{m}^{2}$.

\section{Estimation of neural probes bending force.}


The force, $F$, to deflect a piece of three-layer longitudinal ribbons is estimated by ${ }^{\mathrm{S} 5}$

$$
F=\frac{8 e K_{1 R}}{l^{3}}
$$

Where $e$ is the deflection of the ribbon, $K_{l R}$ is the bending stiffness of the ribbon and $l$ is the length of the ribbon. For the two support arms of the sensors, take $e=10 \mu \mathrm{m}, K_{1 R}=0.54 \times 10^{-15}$ $N \cdot m^{2}$, and $l=200 \mu m . F=2 \times 5.4 n N=10.8 n N$.

Table S1. Key design features of a typical macroporous brain probe are summarized. All features are denoted in Figure S2b-e.

\begin{tabular}{|l|c|c|c|}
\hline Key structural elements & Materials & Dimension & Thickness \\
\hline i. freestanding part of the & SU8 and metal & $6.5 \mathrm{~mm}$, total & Total $\leq 1 \mu \mathrm{m}$ \\
\hline
\end{tabular}




\begin{tabular}{|c|c|c|c|}
\hline probe & & length & \\
\hline $\begin{array}{l}\text { ii. device region of the } \\
\text { probe }\end{array}$ & SU8 and metal & $\begin{array}{l}1 \mathrm{~mm} \text {, total } \\
\text { width }\end{array}$ & Total $\leq 1 \mu \mathrm{m}$ \\
\hline $\begin{array}{l}\text { iii. vertical spacing of the } \\
\text { devices }\end{array}$ & $\mathrm{N} / \mathrm{A}$ & $250 \mu \mathrm{m}$ & $\mathrm{N} / \mathrm{A}$ \\
\hline $\begin{array}{l}\text { iv. longitudinal spacing of } \\
\text { interconnects }\end{array}$ & $\mathrm{N} / \mathrm{A}$ & $50 \mu \mathrm{m}$ & $\mathrm{N} / \mathrm{A}$ \\
\hline \multirow{2}{*}{$\begin{array}{ll}\boldsymbol{v} & \text { longitudinal } \\
\text { interconnects } & \end{array}$} & $\mathrm{Cr} / \mathrm{Au} / \mathrm{Cr}$ & $5 \mu \mathrm{m}$, width & $1.5 / 100 / 1.5 \mathrm{~nm}$ \\
\hline & SU8 & $7 \mu \mathrm{m}$, width & $400 \mathrm{~nm} / \mathrm{metal} / 400 \mathrm{~nm}$ \\
\hline \multirow{2}{*}{$\begin{array}{l}\text { vi. transverse scrolling } \\
\text { elements }\end{array}$} & $\mathrm{Cr} / \mathrm{Pd} / \mathrm{Cr}$ & $3 \mu \mathrm{m}$, width & $10-20 / 80 / 1.5 \mathrm{~nm}$ \\
\hline & SU8 & $10 \mu \mathrm{m}$, width & metal $/ 400 \mathrm{~nm}$ \\
\hline \multirow[t]{2}{*}{ vii. device bend-up arms } & $\mathrm{Cr} / \mathrm{Pd} / \mathrm{Cr}$ & $4 \mu \mathrm{m}$, width & $1.5 / 80 / 30-50 \mathrm{~nm}$ \\
\hline & SU8 & $6 \mu \mathrm{m}$, width & $400 / \mathrm{metal} / 400 \mathrm{~nm}$ \\
\hline \multirow[t]{4}{*}{ viii. sensor metal contact } & $\mathrm{Cr} / \mathrm{Pd} / \mathrm{Cr}$ for $\mathrm{FET}$ & \multirow[t]{2}{*}{$4 \mu \mathrm{m}$, width } & $1.5 / 50-80 / 1.5 \mathrm{~nm}$ \\
\hline & $\mathrm{Cr} / \mathrm{Pt}$ for electrode & & $1.5 / 100 \mathrm{~nm}$ \\
\hline & \multirow[t]{2}{*}{ SU8 } & \multirow[t]{2}{*}{$5 \mu \mathrm{m}$, width } & 400/metal/400 nm, FET \\
\hline & & & $400 \mathrm{~nm} *$, electrode \\
\hline
\end{tabular}

* At electrode sites, only bottom SU8 layer was defined, and the top SU8 layer was absent to expose the electrode contacts. 


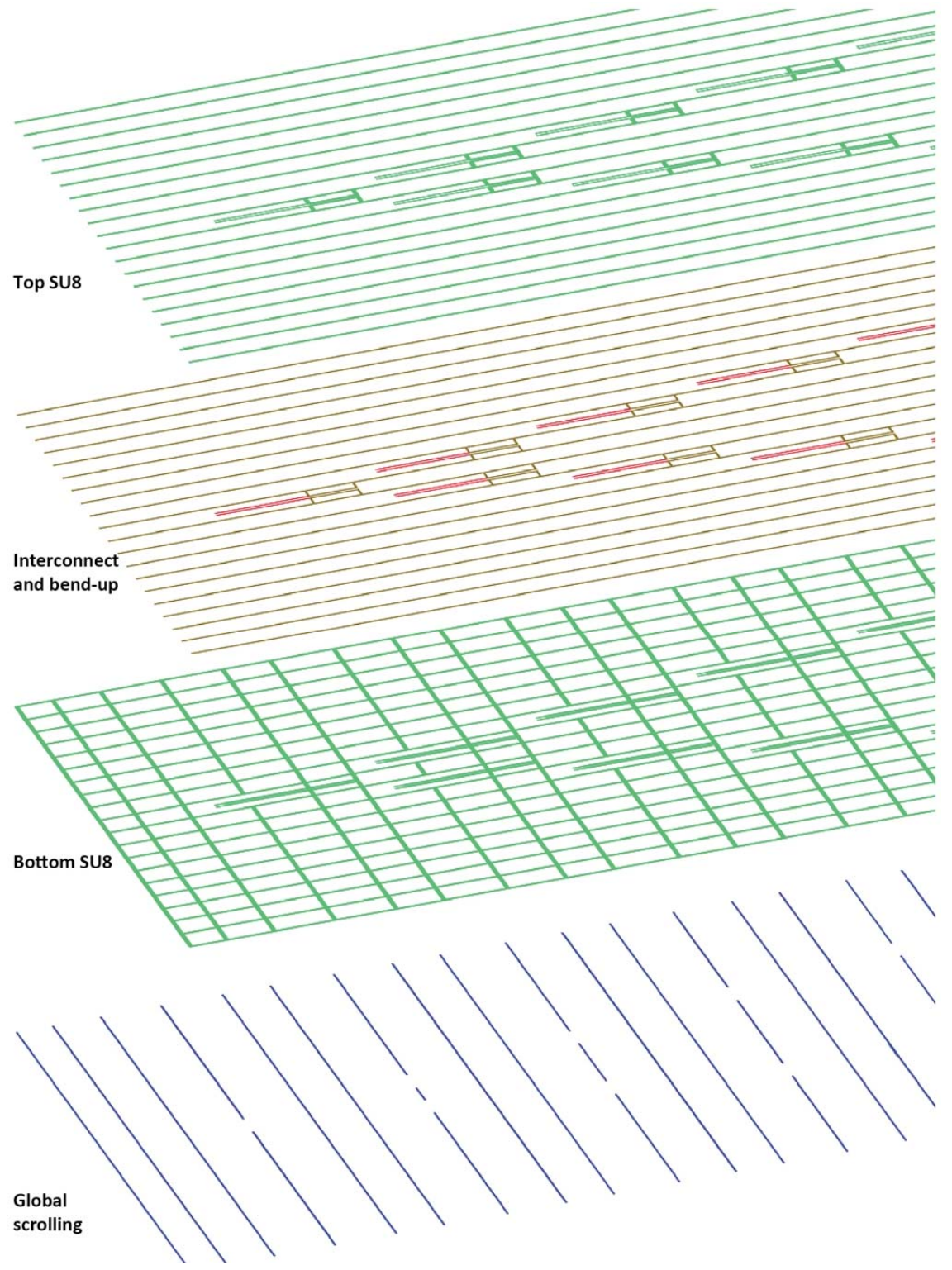

Figure S1. Major layers of a typical macroporous nanoelectronic brain probe design. Bottom-to-top correspond to the sequence defined in our fabrication flow (i.e., starting with global scrolling lines); details of fabrication steps provided in the Supplementary Methods. The purple lines in the global scrolling layer correspond to $\mathrm{Cr} / \mathrm{Pd} / \mathrm{Cr}$ metal ribbons with $10-20 / 80 / 1.5$ $\mathrm{nm}$ thicknesses, respectively, and $3 \mu \mathrm{m}$ widths. Green lines in Bottom and top SU-8 layers indicate $400 \mathrm{~nm}$ thick, $7 \mu \mathrm{m}$ wide SU-8 ribbons. Brown lines in the Interconnects layer represents $5 \mu \mathrm{m}$ wide $\mathrm{Cr} / \mathrm{Au} / \mathrm{Cr}(1.5 / 100 / 1.5 \mathrm{~nm})$ interconnect lines. Red lines in the Bend-up layer arms represent $3 \mu \mathrm{m}$ wide $\mathrm{Cr} / \mathrm{Pd} / \mathrm{Cr}(1.5 / 80 / 30-50 \mathrm{~nm})$ metal lines. 

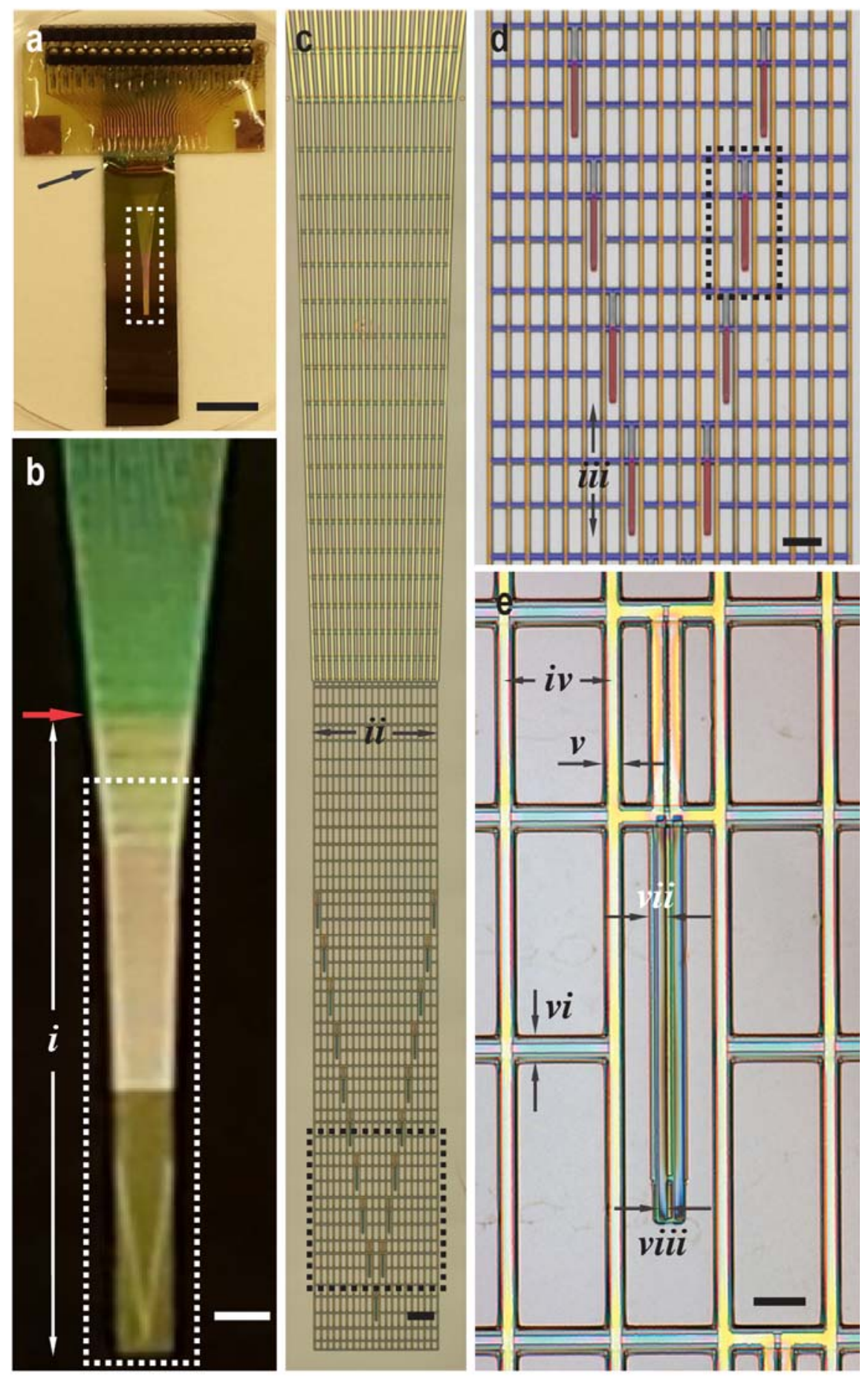
$1 / 3$ of image), with $\mathrm{I} / \mathrm{O}$ to recording instrumentation made by connections to the socket. Electrical connections between the carrier chip and PCB board were made by wire bonding (position highlighted by the black arrow). Scale bar: $1 \mathrm{~cm}$. b, Zoom-in of the mesh electronics area of the probe from the dashed white box in $\mathbf{a}$. The region below the red arrow is on the nickel sacrificial layer. Scale bar: $1 \mathrm{~mm}$. c, Micrograph of the white dashed-box in $\mathbf{b}$, which is released from the substrate following dissolution of the nickel sacrificial layer. The whole probe is designed with an open mesh structure to promote interpenetration and integration with the neural tissue. The individually-addressable sensors are located at design-specified positions in the mesh electronics; for the design shown the sensor elements define two edges of an inverted triangle (bottom 1/3 of the image). Scale bar: $200 \mu \mathrm{m}$. d, Zoomed-in view of the sensor area of the probe (black dashed-box in c.). The strains, which define global scrolling of the mesh electronics and bend-out of the individual sensor elements, are represented by blue and red pseudo-colored regions, respectively. The compressive strain applied in the blue regions generates positive curvature along the transverse direction of the probe, and the tensile strain applied in the red regions generates negative curvature on supporting arm of the sensor (shown in Fig 1c. in main text). Scale bar: $100 \mu \mathrm{m}$. e, Zoomed-in view of an individual sensor element (nanowire FET sensor, position highlighted by the black arrow), outlined by the black dashed box in $\mathbf{d}$. The sensor is located at the tip of the supporting arms. Scale bar: $20 \mu \mathrm{m}$. In the panels, $\boldsymbol{i}$ - viii denote key design elements of the probe; the relevant materials and dimensions of these elements are summarized in Table S1. 


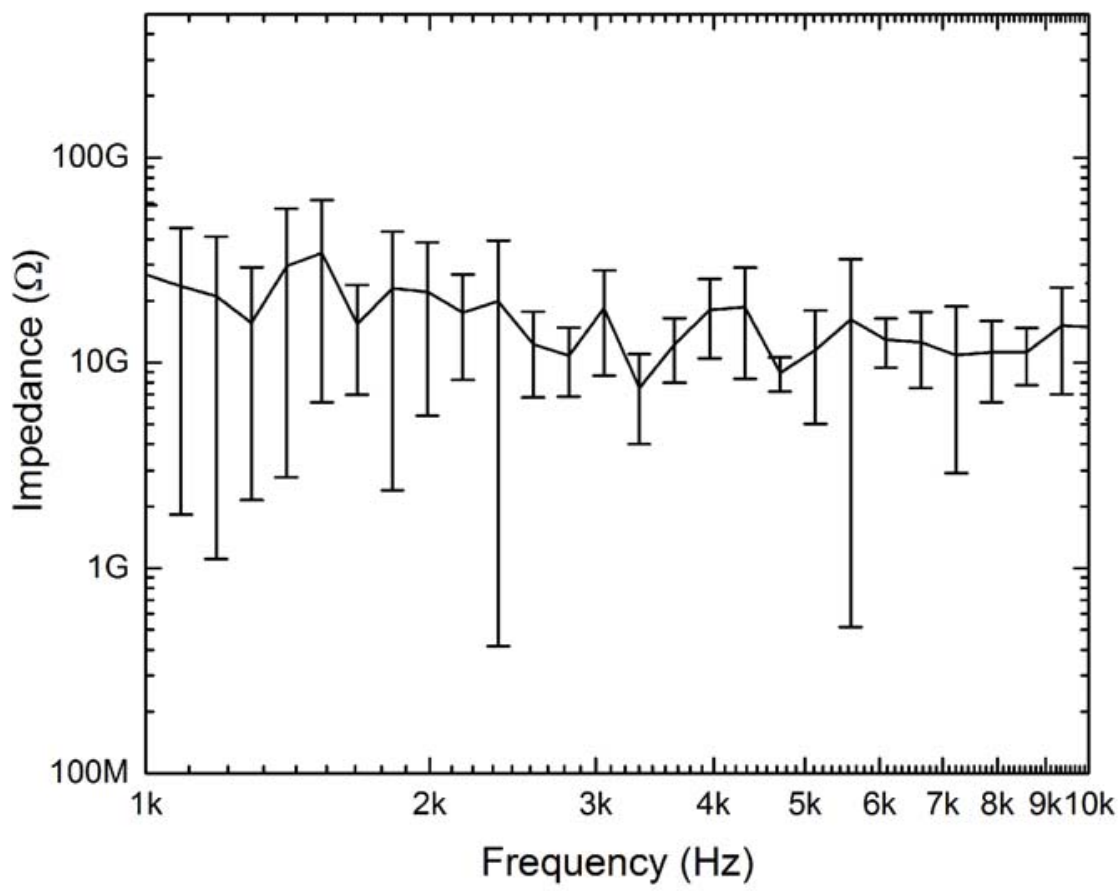

Figure S3. Frequency dependent leakage measurements of the SU8 encapsulated interconnect components. The impedance was measured between 8 pairs of interconnect metal lines in a nanowire FET probe submerged in $1 \mathrm{X}$ PBS, at $100 \mathrm{mV}$ bias and $1-10 \mathrm{kHz}$. The area of each interconnect line in the 1X PBS solution was $2 \mathrm{~mm}$ (length) X $20 \mu \mathrm{m}$ (width). The solid line indicates the average of the measurements and the error bars indicate \pm 1 -standard deviation. 


\section{a}
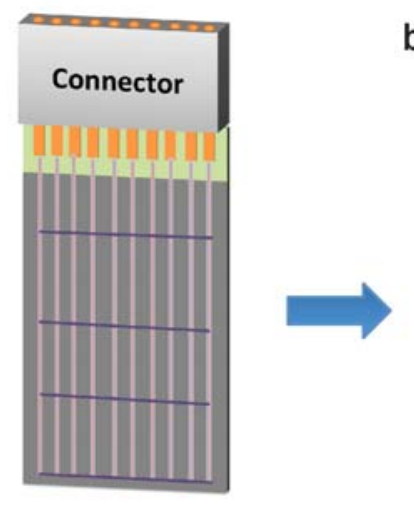

b
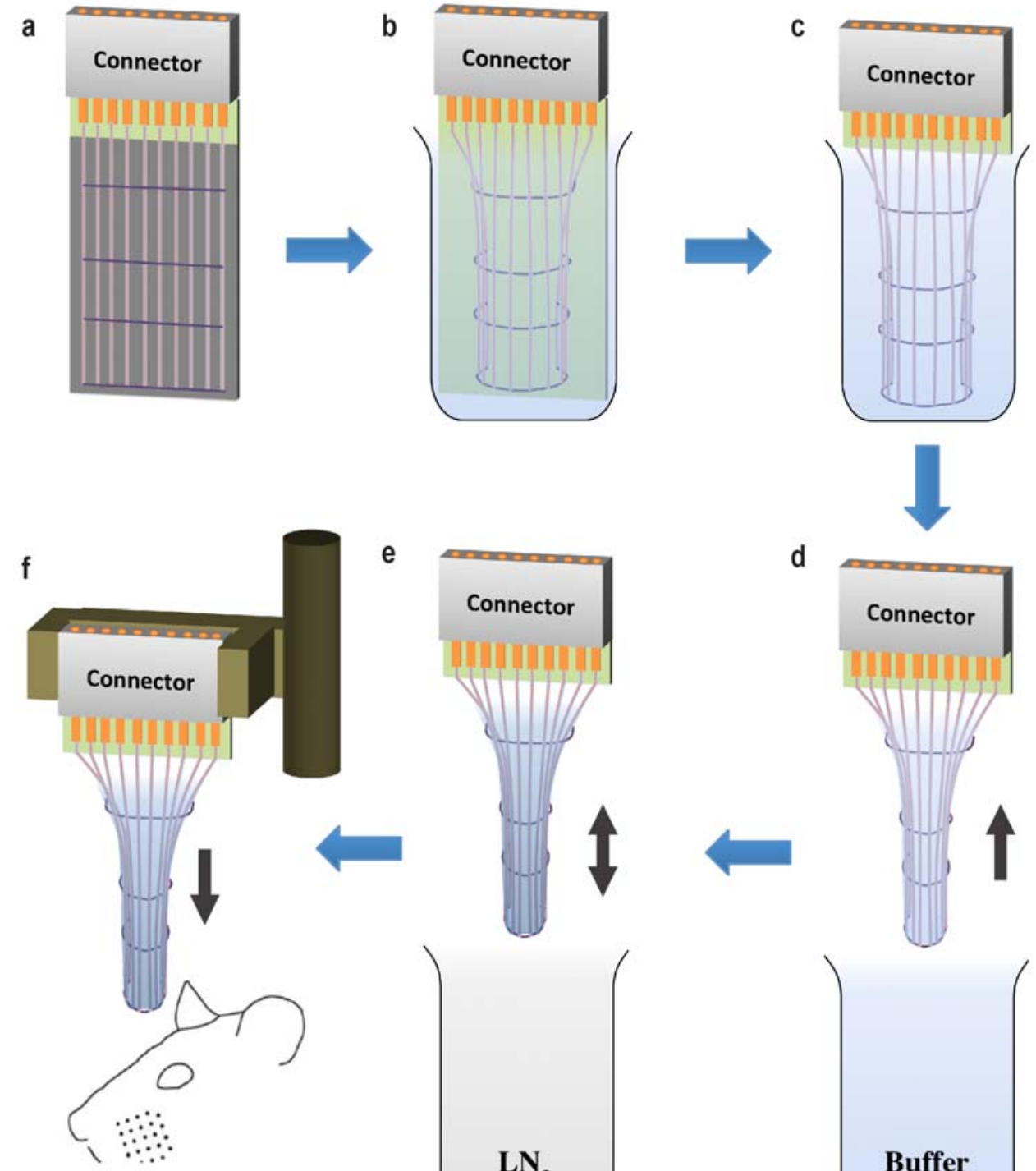

e

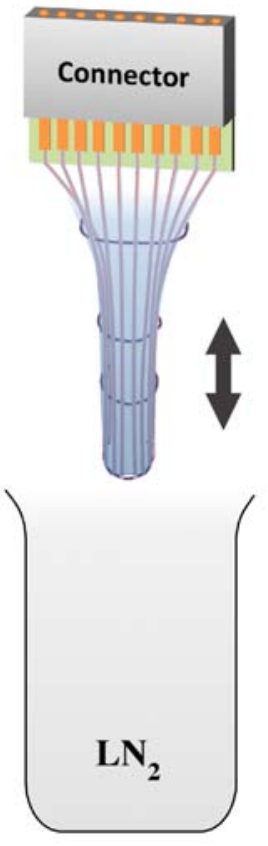

d
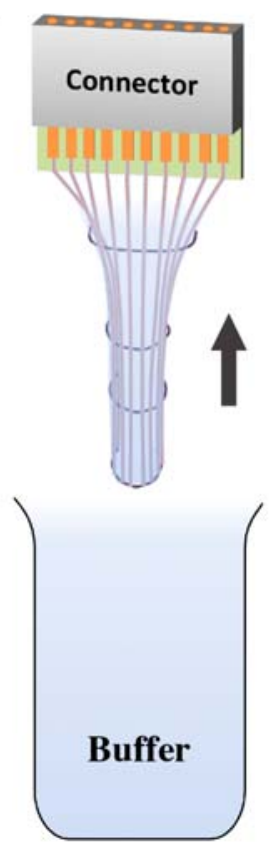

Figure S4. Schematic of probe preparation and frozen insertion. a. The fabricated macroporous neural probe on silicon carrier chip (prior to release) is attached to an $\mathrm{I} / \mathrm{O}$ connector and electrical contacts are made by wire bonding. b. The Ni sacrificial layer is etched and the macroporous neural probe was partially released and freestanding in liquid. c. Excess silicon chip below the connector is trimmed off. d. When the device assembly is removed from the buffer solution, a small amount of buffer is trapped inside the cylindrical probe. Removal was carried out manually with a vertical speed of ca. 1-2 mm/s. e. The probe assembly is then slowly submerged in liquid nitrogen $\left(\mathrm{LN}_{2}\right)$ until ca. thermal equilibrium (5-10 seconds; greatly reduced $\mathrm{LN}_{2}$ boiling). f. The probe is immediately mounted in a custom-holder on a linear translational stage and rapidly inserted into the brain. The insertion step was driven manually with a speed of ca. $5 \mathrm{~mm} / \mathrm{s}$. The entire process should be carried out within $10 \mathrm{~s}$ following removal from the $\mathrm{LN}_{2}$, with the insertion into the brain taking no more than ca. 1 second. 


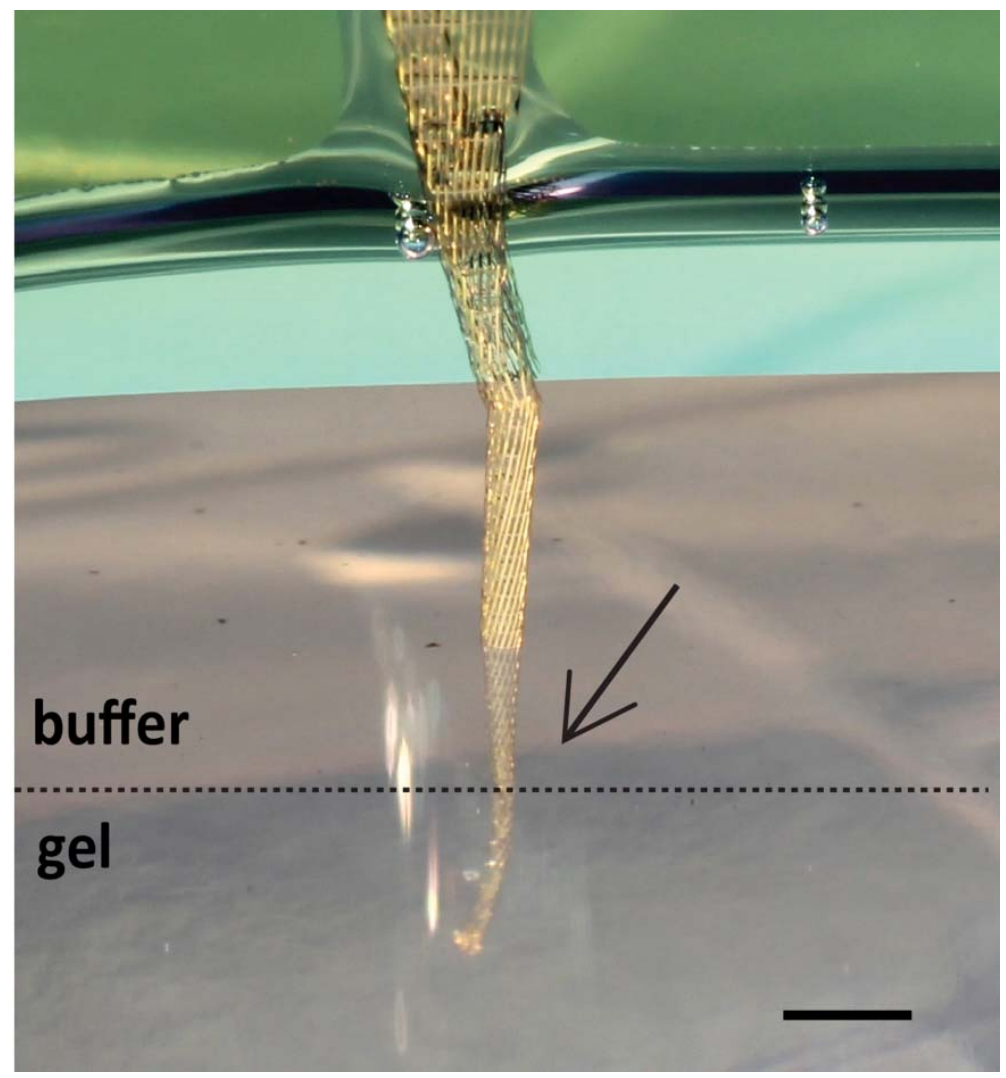

Figure S5. Probe geometry after insertion into hydrogel. Photograph showing a probe tethered to the carrier substrate following partial insertion into $0.5 \%$ agarose hydrogel with ca. 2 $\mathrm{mm}$ buffer solution on top. The $0.5 \%$ agarose gel provides a mechanical resistance during insertion similar to brain tissue ${ }^{\mathrm{S} 6}$. The insertion procedure is the same as discussed in the main text. The probe was frozen by immersion in liquid nitrogen, immediately followed by insertion, which typically took ca. 1 second. The dashed line indicates the gel / buffer boundary, and the arrow points the entry point. Scale bar: $2 \mathrm{~mm}$. 

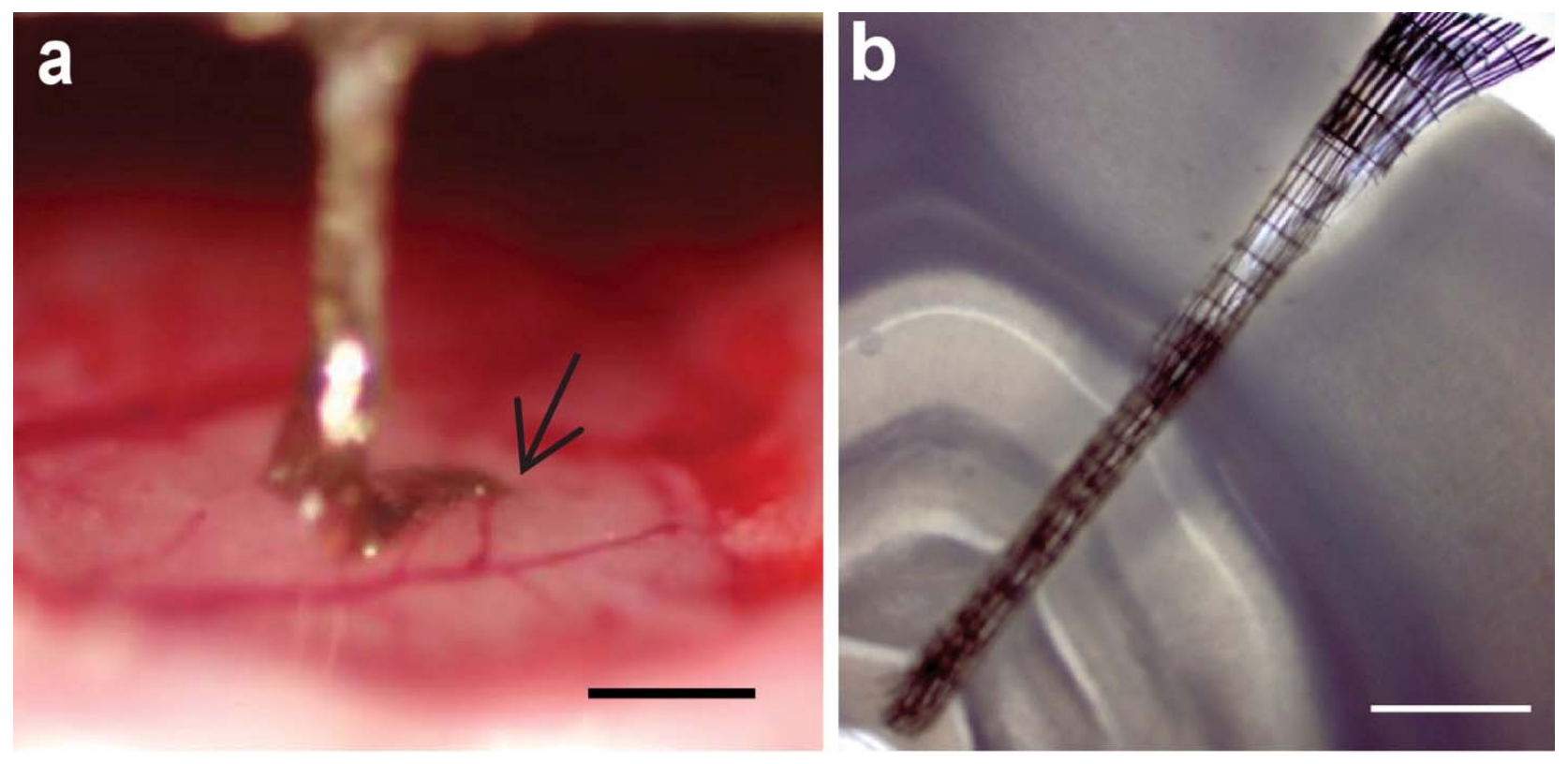

Figure S6. Probe insertion into a rat brain. a, Zoomed-in view of the implantation site in Fig. 3a. The arrow points the small and clean entry site into the brain. The part of the probe outside the rat brain (left of arrow) is relaxed and conformal to the brain surface due to its ultraflexibility. Scale bar: $1 \mathrm{~mm}$. b, Bright field image of a brain slice cut along the probe insertion direction. The brain was fixed and sliced following the procedure described in the Methods within 1 hour after implantation to reveal the probe geometry. The image shows clearly that the probe maintains a straight cylinder shape as designed, thereby yielding a predictable sensor distribution within the tissue. Scale bar: $500 \mu \mathrm{m}$. 

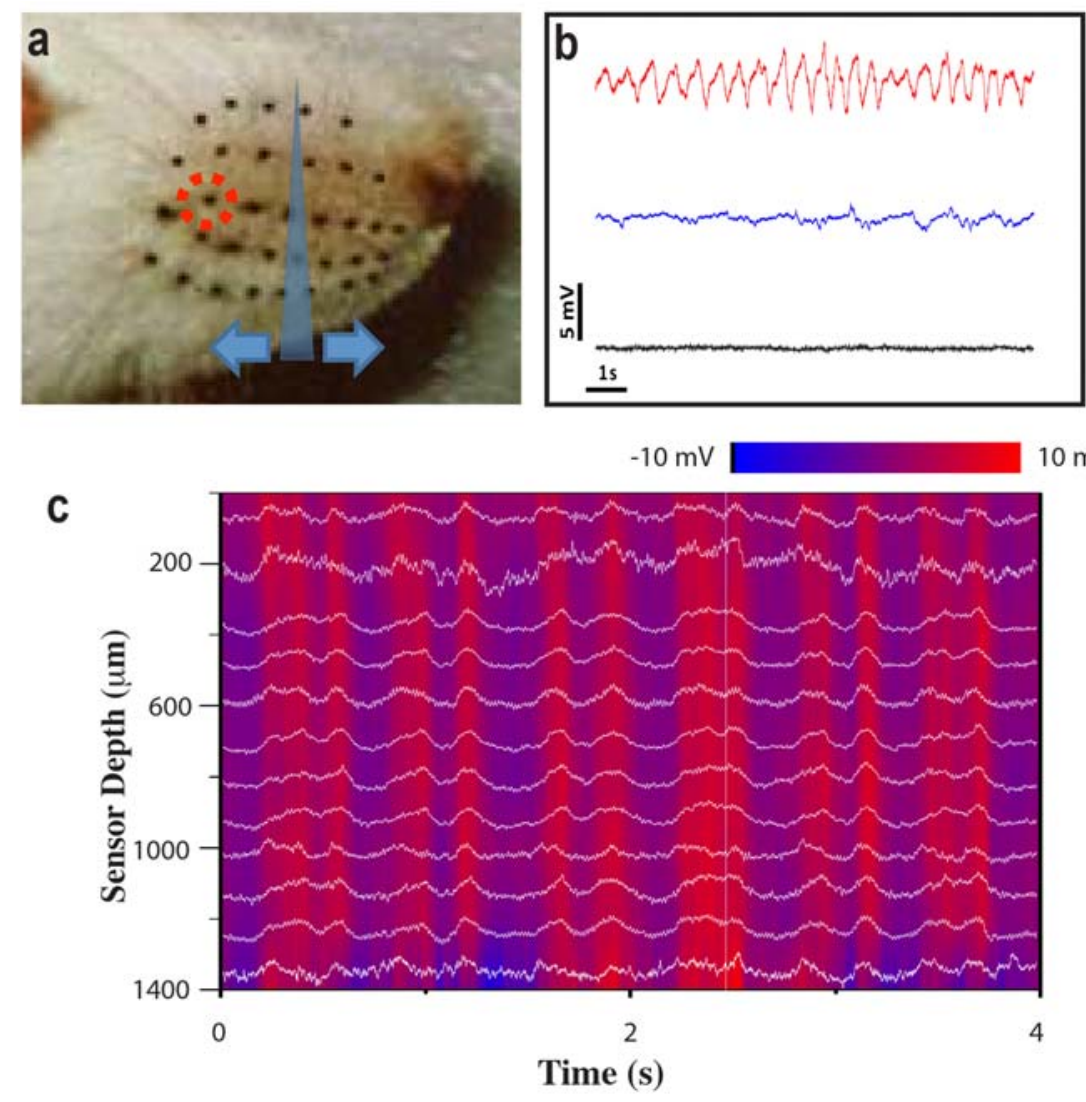

Figure S7. Identification of implanted sub-region of barrel cortex. a. A photograph of whiskers on the contralateral side of the implantation side. Mechanical stimulations were applied to all whiskers to identify the sensor close to a sub-region of barrel cortex. In this implantation and recording, element-2 (Fig. 3b) had the strongest response during stimulation. Subsequently, stimulation of individual whiskers on both contralateral and ipsilateral sides were carried out while simultaneous recording from the implanted mesh probe. We observed that only stimulations applied to the $\mathrm{C} 1$ whisker (indicated by dashed circle) on the contralateral side could elicit a strong response from element- 2 of the probe. b, Three representative recordings from element-2. Red, while stimulating $\mathrm{C} 1$ whisker; blue, while stimulating the adjacent whisker C2; black, while stimulating whisker $\mathrm{C} 1$ after the rat was euthanized. c, A 4-second map of the multiplexed LFP recording from the same experiment as shown in Fig. 3c. The vertical axis represents the depth beneath the brain surface. The horizontal axis indicates the recording time. Colors highlight the amplitude of the recorded LFPs. 

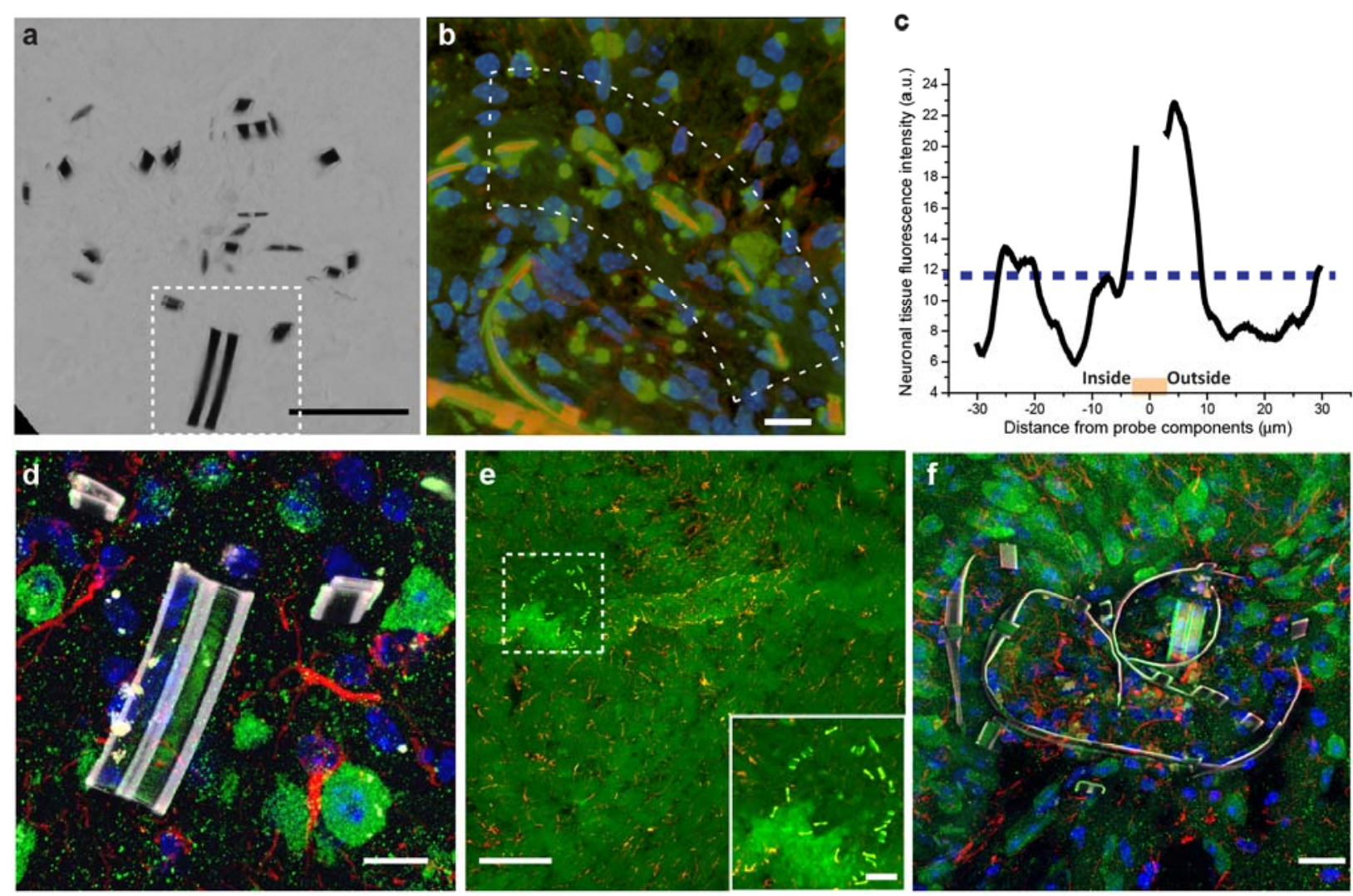

Figure S8. Histology of the probe-tissue interface. a. Bright field images of a $20 \mu \mathrm{m}$ thick slice showing the interface 5 weeks post implantation. The brain tissue has interpenetrated the probe with time (compared with acute cross-section images (e.g., Fig. 4b). Dashed box indicates a pair of supporting arms with a sensor at the end. Scale bar: $50 \mu \mathrm{m}$. b, The reconstructed confocal micrograph of immunochemically labeled cross-section slice shown in Fig. 4e, where the curved dashed box here encompasses tissue both inside and outside the indicated the curved probe surface. The pseudo color-coding is as follows. Blue: nucleus stained with Hoechst; green: neurons stained with $\beta$-tubulin-III; orange: SU-8, and red: GFAP. Scale bar: $10 \mu \mathrm{m}$. The fluorescence intensity in the dashed area is used to analyze the affinity of neurons to probe components. c, The average neuron fluorescence intensity from green channel ( $\beta$-tubulin-III, neuronal tissue) along the short axis of the outlined area in $\mathbf{b}$ is plotted, from inside of the probe to outside. The orange line indicates the position of probe. The blue dashed line indicates the average fluorescence intensity of the green channel in the whole image. These results demonstrate there is a higher density of neurons near the probe components, and thus suggest a tendency of neurons to form tight junctions with the probe components. The tissue slices were 
prepared 5-weeks post implantation into the somatosensory cortex region of a mouse (Methods). d. Zoom-in view of a bend-up nanowire sensor in Fig. 4c (indicated as dashed box area in a.) illustrating the proximity of sensor arms and neurons as well as the intact SU-8/metal/SU-8 structure. Scale bar: $10 \mu \mathrm{m}$. e. A reconstructed confocal micrograph of immunochemically labeled cross-section from the same mouse brain and brain probe sample as used in Fig. 4C. Scale bar: $100 \mu \mathrm{m}$. Inset: Zoom-in view of the white boxed region. Scale bar: $20 \mu \mathrm{m}$. The sample was ca. 5 slices $/ 120 \mu \mathrm{m}$ deeper in the brain relative to Fig. $4 \mathrm{C}$; the same staining and imaging methods were used for both samples. f. A "collapsed" probe resulting from slow implantation of the frozen probe (i.e. insertion time of the probe in the brain tissue longer $>1 \mathrm{~s}$ ). The probe becomes randomly folded due to thawing before the insertion was completed. This result demonstrates the importance of rapid insertion of the frozen probes to maintain the designed geometry. Although the probe did not hold the designed geometry, this probe also showed tight integration with neurons, which is consistent with the macroporous ultra-flexible nature of the structure. The tissue slices were prepared 5-weeks post implantation into the somatosensory cortex region of a mouse using the same the same staining method as in in Fig. 4C (Methods). Scale bar: $20 \mu \mathrm{m}$. 


\section{Supplementary References}

S1. Patolsky, F., Zheng, G. \& Lieber, C. M. Fabrication of silicon nanowire devices for ultrasensitive, label-free, real-time detection of biological and chemical species. Nat. Protoc. 1, 1711-1724 (2006).

S2. Dellas, N. S., Schuh, C. J. \& Mohney, S. E. Silicide formation in contacts to Si nanowires. J Mater Sci 47, 6189-6205 (2012).

S3. Lee, K. N., et al. Stress-induced self-rolled metal/insulator bifilm microtube with micromesh walls. J Micromech Microeng 23 (2013).

S4. Javey, A., Nam, S., Friedman, R. S., Yan, H. \& Lieber, C. M. Layer-by-layer assembly of nanowires for three-dimensional, multifunctional electronics. Nano Lett. 7, 773-777 (2007).

S5. Steif, P. S. Mechanics of materials. Pearson: Upper Saddle River, NJ, 2012.

S6. Sharp, A. A., Ortega, A. M., Restrepo, D., Curran-Everett, D. \& Gall, K. In vivo penetration mechanics and mechanical properties of mouse brain tissue at micrometer scales. IEEE Trans. Biomed. Eng. 56, 45-53 (2009). 